\title{
A study of the medicinal plants used by the Marakwet Community in Kenya
}

Wilson Kipkore ${ }^{1}$, Bernard Wanjohi ${ }^{2}$, Hillary Rono ${ }^{3}$ and Gabriel Kigen ${ }^{4 *}$

\begin{abstract}
Background: The medicinal plants used by herbalists in Kenya have not been well documented, despite their widespread use. The threat of complete disappearance of the knowledge on herbal medicine from factors such as deforestation, lack of proper regulation, overexploitation and sociocultural issues warrants an urgent need to document the information. The purpose of the study was to document information on medicinal plants used by herbalists in Marakwet District towards the utilization of indigenous ethnobotanical knowledge for the advancement of biomedical research and development.

Methods: Semi- structured oral interviews were conducted with 112 practicing herbalists. The types of plants used were identified and the conditions treated recorded.

Results: Herbal practice is still common in the district, and 111 plants were identified to have medicinal or related uses. Different herbal preparations including fruits and healing vegetables are employed in the treatment of various medical conditions. Veterinary uses and pesticides were also recorded.

Conclusion: The study provides comprehensive ethnobotanical information about herbal medicine and healing methods among the Marakwet community. The identification of the active ingredients of the plants used by the herbalists may provide some useful leads for the development of new drugs.
\end{abstract}

Keywords: Marakwet, Herbal medicine, Ethnobotanical, Plants, Documentation, Research

\section{Background}

Medicinal plants have been used by humans from time immemorial. Many drugs have plant origin, and several plants are currently undergoing investigation to ascertain their therapeutic efficacies [1-3]. Traditional herbal medicine is still an important component of healthcare in sub-Saharan Africa. This is largely due to poverty, inadequacy of health services and shortage of health workers. Even when the facilities exist, there is rampant shortage of drugs and equipment. The World Health Organization (WHO) estimates that up to $80 \%$ of the population in some developing countries use traditional medicine $[4,5]$.

Like many other developing countries, the use of traditional herbal medicine is still widespread in Kenya, especially in the rural areas [4]. In some instances, herbal remedies are combined with conventional medicine especially when the subjects feel that the prescription drugs

\footnotetext{
*Correspondence: kigengfk@gmail.com

${ }^{4}$ Department of Pharmacology and Toxicology, Moi University School of Medicine, P.O. Box 4606, Eldoret, Kenya

Full list of author information is available at the end of the article
}

are not effective [6]. Despite this, most of the ethnobotanical information on herbal medicine and healing methods largely remain undocumented. This is compounded by the fact that in most Kenyan communities, the information is passed on orally, and only to very close relatives who might not necessarily be interested in practicing the art [7]. Lack of proper regulation has also led to the emergence of quack herbalists. The wanton destruction of forests and use of modern medicine have also contributed to the risk of the information disappearing completely over time [8]. There is therefore urgent need to document the knowledge on herbal information used by different communities in Kenya [7].

The Marakwet are a sub-tribe of the larger Kalenjin community residing in Rift Valley region. The other members of the Kalenjin ethnic group include Kipsigis, Nandi, Keiyo, Tugen, Pokot, Terik and Sabaot. They are Highland Nilotes, and are culturally related to the Maasai and Samburu. Apart from the Sabaot, all Kalejins live in the Great Rift Valley. The Sabaot live around Mt Elgon 
and are therefore citizens of both Kenya and Uganda. They are excellent athletes, and provide the vast majority of the Kenyan and of late Ugandan athletes with international honours in long distance races [9-11]. The Marakwet are mainly semi-pastoral just like the other Kalenjins [12]; Among the Kalenjin community, Marakwet District is one of the regions with a considerable number of authentic practicing herbalists. This is unlike many other regions of Kenya where there has been rapid decline in the use of herbal medicine. The local herbalists complement conventional local doctors in the treatment of common diseases within the district.

The perception of disease among the Marakwet like many other African Communities, is a complex matter. Disease or illness is thought to be associated with pollutants, misfortune, curse, "Bon" (witchcraft or sorcerers), or people with "bad eyes;" "bich cho tinye konyen". In most cases, these people (both men and women) are not even aware that they have "bad eyes". Usually they discover with horror that they can cause harm without their intention by merely looking at an object or person [13]. Each disease is therefore categorized according to the source, and the treatment differs. The practice of sorcery is not very common among the Marakwet. Treatment is by witchdoctors who use several methods including sacrificial animals. Preventive charms are used to protect against witches and people with "bad eyes". Disease causing pollutants are treated by herbalists [13]. Pollutants, which are thought to emanate from such conditions as changes in weather; poisoning, insect bites, food and sex are treated by use of herbs. Currently, traditional herbal medicine is still viewed as complimentary to modern medicine. Amongst the Marakwet and their Keiyo counterparts, mental illness, infertility in women, chronic diseases such as diabetes and fractured skulls are reserved for traditional healers, whereas diseases such respiratory diseases including, pneumonia, fever and accidents are treated by modern medicine $[13,14]$.

The use of Ethnobotanical medicines has been part and parcel of the health system of the Marakwet community from time immemorial. It has had profound socio-economic, cultural and educational values, which in turn has provided opportunities for enhancing their livelihoods within the Greater Rift Valley [13]. Historically, the Marakwet had an integrated health system that comprised of both herbal medicine and surgery. The herbalists treated common ailments, whereas the surgeons performed simple, and sometimes complicated surgical procedures such as craniotomy and tonsillectomy. The only other Kenyan communities on record to have practiced this form of specialized surgery are the Kisii, Meru and Kuria [14,15].

However most of the herbal preparations have not been recorded. Dr Lindsay, a missionary who worked at
Kapsowar Mission Hospital for over 20 years published a book on Medicinal plants used by the Marakwet people which was however not exhaustive [16]. This is largely due to the fact that the practice is in most cases a closely guarded family secret. For instance, herbalists from the same locality may use similar plants to treat different conditions. Also, being a foreigner and missionary, Dr Lindsay faced several challenges in convincing the herbalists to reveal the herbs despite his long stay in the region. This was in part attributed to the negative approach by colonialists to traditional medicine who associated traditional healers with witchcraft or "black magic" and superstition [17]. Most of the herbalists with whom he interacted with are those who had converted to Christianity, mainly residing in the highlands around Kapsowar. Some few Marakwet herbal medicinal plants have also been reported by Kokwaro [18]. There are also published reports about traditional herbal medicine which have been carried out among the other Kalenjin tribes, notably the Nandi and Sabaot [19-21].

\section{Materials and methods Study area}

The study was conducted within Marakwet district, which is located in the North rift Valley region of Kenya (Figures 1, 2). The district, together with Keiyo, form Elgeyo Marakwet County [22]. The district is situated between $0^{\circ} 51^{\prime}$ to $1^{\circ} 19^{\prime} \mathrm{N}$ and $35^{\circ} 29^{\prime}$ to $35^{\circ} 43^{\prime} \mathrm{E}$, and covers a total area of $1588 \mathrm{Km}^{2}$ (Statoids). It has two regions; the highland also referred to by the residents as "Mosop"; and lowland located on the floor of Kerio Valley also known as "Kew" or "Endo". In between the two regions is the escarpment, a long line of steep slopes consisting of cliffs separating the lowland and highland areas; with distinct vegetation type (Figure 2).

The highland region rises up to $3300 \mathrm{~m}$ above sea level at the highest point (Cheran'gany hills), and is mainly forested with low temperatures of about $13^{\circ} \mathrm{C}$. The district is one of the most forested in the country, with natural forests covering $43 \%$ of the land, a total of 65, 000 hectares [24]. In addition, unlike other regions of Kenya such as the Mau forest, it has relatively not suffered heavy deforestation, and is a source of many streams which feed Lake Victoria in the Western part, and Lake Turkana in the East [23-25]. Cherangani Hills is part of the five main closed forests areas in Kenya that are protected by the government. The others are the Aberdares, Mount Kenya, Mount Elgon and South West Mau [26,27]. Although still heavily forested, Cherangani now faces risk of serious degradation due to illegal forest clearing $[23,24,28]$. The escarpment region is about $1000 \mathrm{~m}$, and is part of the Great Rift Valley. It is mainly semi arid with temperatures rising up to $35^{\circ} \mathrm{C}$. 


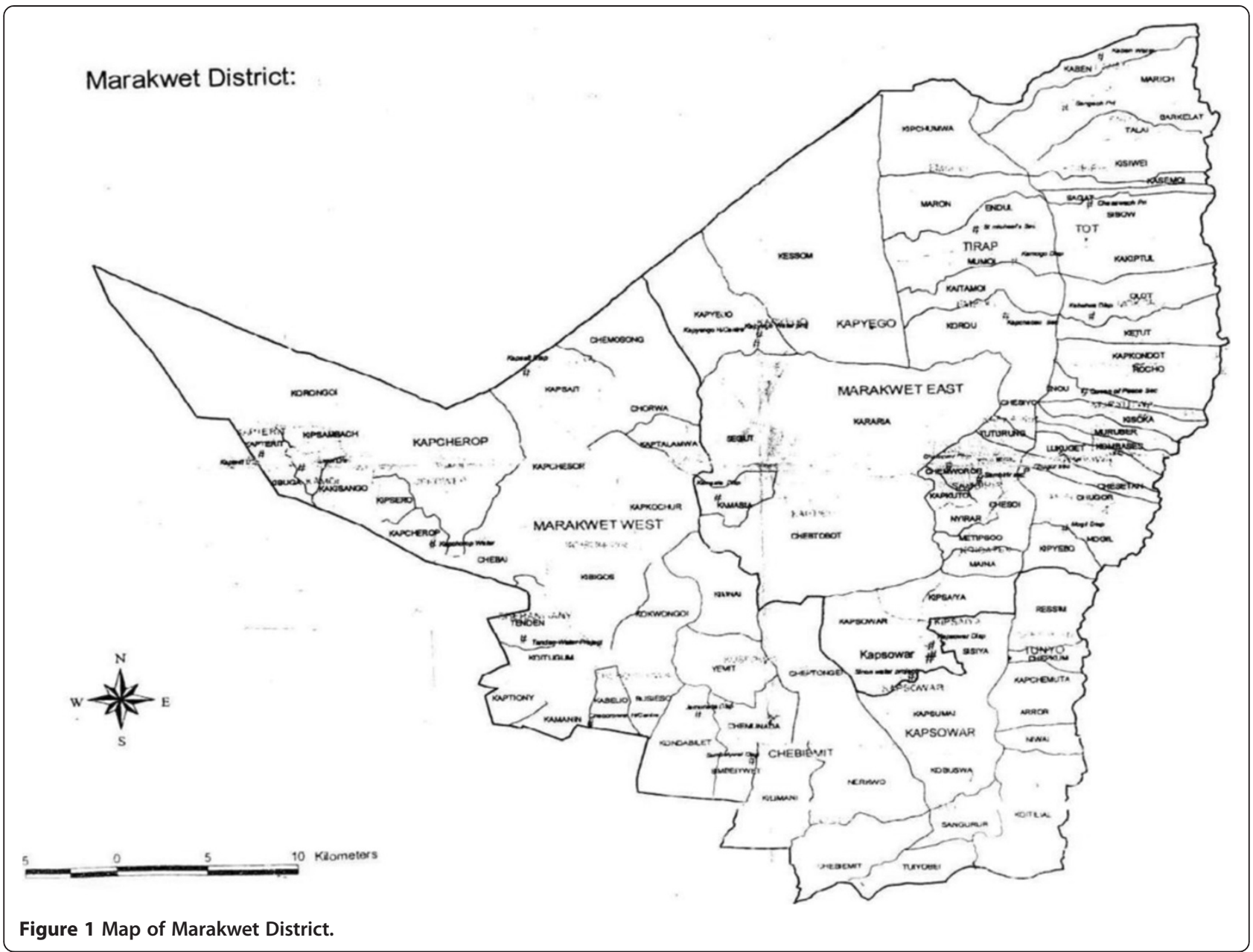

The geographical landscape provides a uniquely wide topography, with a rich biodiversity comprising of different plant species, and in some instances largely undisturbed indigenous forests. The plant biodiversity in the Valley is particularly luxuriant and varied especially on the escarpment. Stunted trees, shrubs, succulents (Sanseviera spp.), and myriad of plants grow here in profusion. The herbalists use the numerous plants found throughout the diverse ecosystems from the Kerio Valley to the highlands $[23,24]$. Information on the most prevalent diseases within the district was obtained from the Medical officer of Health office. The population per doctor ratio in Elgeyo Marakwet County is 62,000: 1 [22,29].

\section{Data collection}

The research team comprised of a group of professionals from both the medical field and plant specialists led by four scientists; a plant specialist (Kipkore), taxonomist (Wanjohi), a pharmacologist (Kigen) and an ophthalmologist (Rono). Apart from their respective professions, Kipkore and Kigen are residents of Elgeyo Marakwet County, and could therefore easily communicate in the local dialect. Rono has been involved in Trachoma control programs within the county, and is well known in the District. Ethnobotanical survey was conducted in different parts of Marakwet district over a 12-month period between February 2011 and January 2012.

Reconnaissance visits were initially conducted in order to identify the known/authentic herbalists in the district. The initial selection was based on the willingness of herbalists to voluntary give information and interaction with the researchers during consultative meetings. These meetings were participatory in nature, with acquaintances from the local communities as facilitators. Local opinion leaders including Church and village elders, Chiefs and Councilors were enlisted to assist in the identification, and to convince the herbalists to volunteer the information. Formal interviews were avoided. This was crucial as the herbalists had to clearly understand our motives before providing information, since most of the practicing herbalists were old and uneducated.

Ethnobotanical data was collected from the herbalists, both men and women practicing across the district. Their age ranged from 35 to 76 years, with the number 


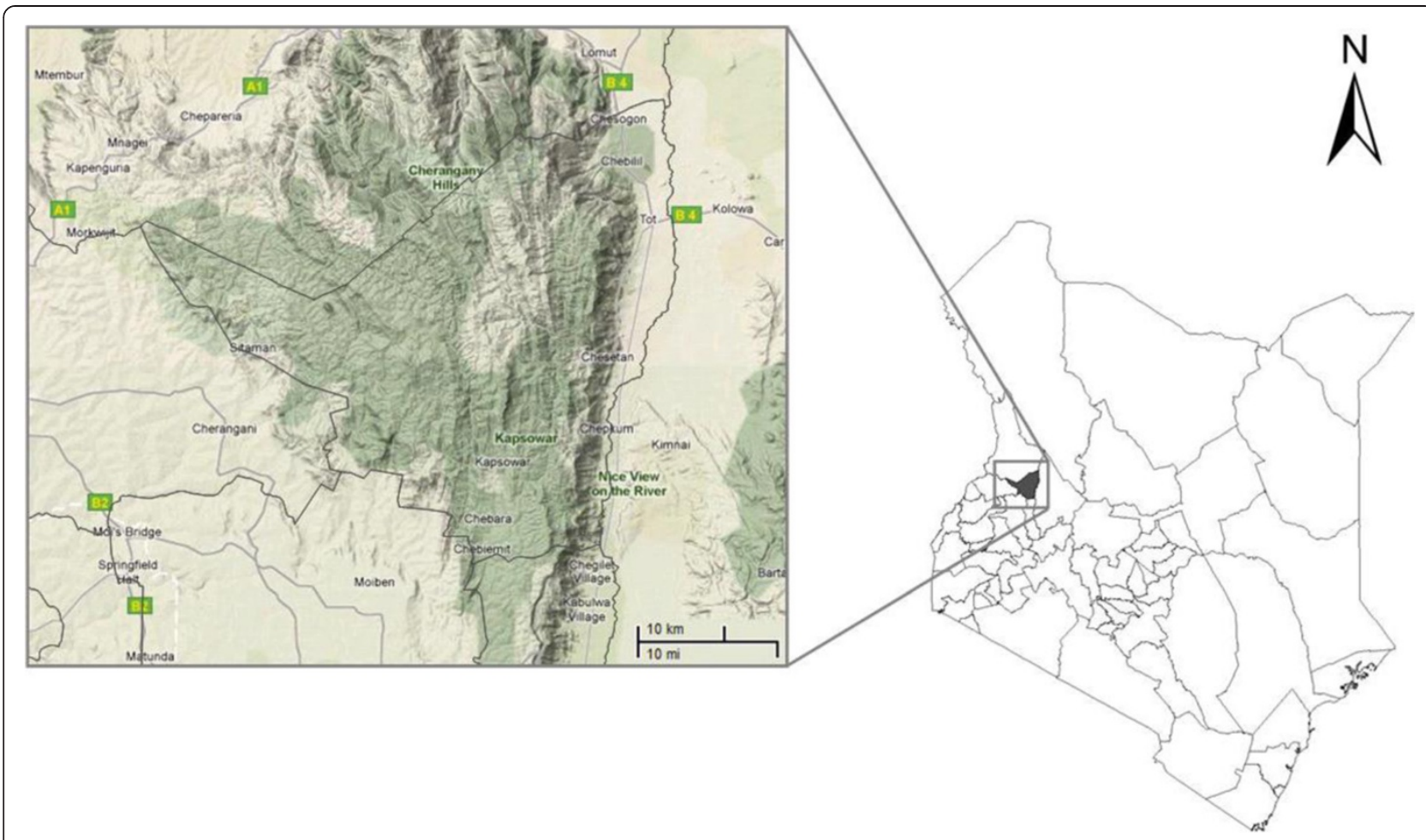

Figure 2 Terrain map of Marakwet district on the left, and the Map of Kenya showing the position of Marakwet district in the right. (c) 2010 Google - Map Data $\odot 2010$ Google, Tracks4Africa. Adapted from [23].

of female herbalists being $74(66 \%)$ and their male counterparts 40(38\%). The herbalists were interviewed within their practice, which in most cases was their residence, or Market centres. After that, the interviewees were requested to accompany the authors to the field to identify the plants. The plants were then photographed and collected. Where possible, the patients were also interviewed in order to corroborate the evidence of their treatment with the claims by the herbalists. The medicinal plants used in healthcare, herbal drugs preparations, local/botanical names; and the diseases treated were recorded. A total of 112 herbalists, all being Marakwets were identified, and all were included the study. The collected plants were identified by Kipkore and Wanjohi and all voucher specimens were deposited at the University of Eldoret Botanical Herbarium, for future reference. Kigen and Rono interviewed both the herbalists and patients, in order to identify the illnesses treated. Nomenclature of trees, shrubs and lianas were as per "Kenya Trees Shrubs and Lianas" [30], while the herbs were named according to "Kenya Upland Wild Flowers" [31]. The data was then compared to that from the previous studies that have been carried out in the region.

\section{Results and discussion}

A total of 111 medicinal plants species were collected, out of which 3 could not be identified. The list of the plants and their respective uses are as outlined in Table 1. The 108 identified plants belonged to of 53 families. This compares well to previous studies that were done in $\mathrm{Mt}$ Elgon, where the other Kalenjin, the Sabaot reside [21], but significantly higher than that of their Nandi cousins [20]. This could be attributed to the diverse ecosystem in both Marakwet and Mt Elgon regions, and the fact that herbal practice is still widespread in the two districts.

The family with the highest number of reported medicinal plant species was Mimosaceae 8(7.4\%), followed by Euphorbiaceae 7(6.5\%) and Asteraceae, 6(5.6\%) (Table 2). Mt Elgon study had Fabaceae, Euphorbiaceae and Asteraceae while Nandi had Acanthaceae, Asteraceae and Amaranthaceae in the same order [20,21]. Of the three unidentified plants, we were informed that "Turesio" which is a large tree, has been overharvested for making cooking sticks and building poles. We also got to learn that "Seremwo", also a tree grows in areas of the escarpment that are difficult to access, while "Mindililwo ne mining" is an ephemeral that only grows for a short period during the rainy season. Most of the medicinal plants used either grew on the lowlands or escarpment region. Table 3 shows the distribution of the plants as per region. Trees were most widely used plant parts $41(37 \%)$ followed by herbs $32(28 \%)$ and shrubs $29(26 \%)$. Only nine species $(8 \%)$ were climbers (both liana and shrubs) (Table 4). All the plants were reported by their local names. 
Table 1 List of the various Herbal plants used by herbalistsand corresponding uses

\begin{tabular}{|c|c|c|c|c|c|c|c|c|c|}
\hline Botanical name & Voucher no. & Local name & Common name & Family & Habit & Habitat & Parts used & Method & General plant uses \\
\hline $\begin{array}{l}\text { Acacia brevispica } \\
\text { Harms }\end{array}$ & WBHG/13/081 & Korniswo & Prickly Thorn Bonsai & Mimosaceae & Shrub & $\begin{array}{l}\text { Lowland/ } \\
\text { escarpment }\end{array}$ & Leaves & $\begin{array}{l}\text { Crushed and rubbed } \\
\text { on skin }\end{array}$ & Itchy skin rashes \\
\hline $\begin{array}{l}\text { Acacia brevispica } \\
\text { Harms }\end{array}$ & WBHG/13/081 & Korniswo & Prickly Thorn Bonsai & Mimosaceae & Shrub & $\begin{array}{l}\text { Lowland/ } \\
\text { escarpment }\end{array}$ & Leaves & Crused and applied & Removal of ganglion \\
\hline $\begin{array}{l}\text { Acacia hockii } \\
\text { De. Willd. }\end{array}$ & WBHG/13/026 & Churur & $\begin{array}{l}\text { Whistling thorn/ } \\
\text { shittim wood }\end{array}$ & Mimosaceae & Tree & Escarpment & Bark & Boiled and administered & Abdominal (colic) pains \\
\hline $\begin{array}{l}\text { Acacia hockii } \\
\text { De. Willd. }\end{array}$ & WBHG/13/026 & Churur & $\begin{array}{l}\text { Whistling thorn/ } \\
\text { shittim wood }\end{array}$ & Mimosaceae & Tree & Escarpment & Bark & Boiled with water & Herbal "tea" \\
\hline $\begin{array}{l}\text { Acacia lahai [Steud. \& } \\
\text { Hochst. ex] Benth }\end{array}$ & WBHG/13/038 & Seretetwo & Red thorn & Mimosaceae & Tree & Highland & Leaves & $\begin{array}{l}\text { Pounded and mixed } \\
\text { with other herbs }\end{array}$ & Synergistic herb \\
\hline $\begin{array}{l}\text { Acacia mellifera } \\
\text { (Vahl) Benth }\end{array}$ & WBHG/13/043 & Ngowo & $\begin{array}{l}\text { Black thorn/ } \\
\text { wait-a-bit thorn }\end{array}$ & Mimosaceae & Shrub & Lowland & Roots & Boiled and administered & $\begin{array}{l}\text { Sexually transmitted } \\
\text { diseases }\end{array}$ \\
\hline $\begin{array}{l}\text { Acacia mellifera } \\
\text { (Vahl) Benth }\end{array}$ & WBHG/13/043 & Ngowo & $\begin{array}{l}\text { Black thorn/ } \\
\text { wait-a-bit thorn }\end{array}$ & Mimosaceae & Shrub & Lowland & Higher parasites & Burnt and ashes licked & Rheumatism \\
\hline $\begin{array}{l}\text { Acacia nilotica } \\
\text { (L.) Delile }\end{array}$ & WBHG/13/068 & Ngopkwo & $\begin{array}{l}\text { Thorntree/whistling } \\
\text { thorn }\end{array}$ & Mimosaceae & Tree & $\begin{array}{l}\text { Lowland/ } \\
\text { escarpment }\end{array}$ & Bark & $\begin{array}{l}\text { Bark chewed when } \\
\text { raw or boiled }\end{array}$ & Abdominal (colic) pains \\
\hline Acacia tortilis Hayne & WBHG/13/039 & Sesia & Shittim wood & Mimosaceae & Tree & $\begin{array}{l}\text { Lowland/ } \\
\text { escarpment }\end{array}$ & Fruit bodies & Burnt and ash licked & Cancer \\
\hline $\begin{array}{l}\text { Acalypha fruticosa } \\
\text { Forsk. }\end{array}$ & WBHG/13/101 & Sawiyon & $\begin{array}{l}\text { Birch-leaved } \\
\text { Acalypha }\end{array}$ & Euphorbiaceae & Herb & $\begin{array}{l}\text { Lowland/ } \\
\text { escarpment }\end{array}$ & Leaves & $\begin{array}{l}\text { Crushed and applied } \\
\text { on the site }\end{array}$ & Scorpion/bee stings \\
\hline $\begin{array}{l}\text { Acokanthera } \\
\text { schimperi (A. DC.) } \\
\text { Benth. \& Hook. }\end{array}$ & WBHG/13/110 & Kelyo & Arrow-poison plant & Apocynaceae & Tree & Escarpment & Roots & Boiled and applied & Arrow poison \\
\hline Albizia spp. & WBHG/13/063 & Seet & Flea tree /frywood & Mimosaceae & Tree & Escarpment & Fruit bodies & Burnt and ash licked & Cancer \\
\hline Albizia spp. & WBHG/13/063 & Seet & Flea tree /frywood & Mimosaceae & Tree & Escarpment & Bark & Boiled and administered & $\begin{array}{l}\text { Abortifacient, } \\
\text { Contraceptive }\end{array}$ \\
\hline $\begin{array}{l}\text { Albuca bracteata } \\
\text { (Thunb.) J.C. } \\
\text { Manning \& } \\
\text { Goldblatt }\end{array}$ & WBHG/13/049 & Kdow & $\begin{array}{l}\text { Pregnant onion } \\
\text { plant }\end{array}$ & Asparagaceae & Herb & Lowland & Bulbs & Boiled and administered & Cancer \\
\hline Aloe spp. & WBHG/13/095 & Chepkenderetwo & Burn plant & Aloaceae & Herb & $\begin{array}{l}\text { Lowland/ } \\
\text { escarpment }\end{array}$ & Leaves & Crushed and sap applied & Wounds \\
\hline Aloe spp. & WBHG/13/095 & Chepkenderetwo & Burn Plant & Aloaceae & Herb & $\begin{array}{l}\text { Lowland/ } \\
\text { escarpment }\end{array}$ & Leaves & $\begin{array}{l}\text { Sap applied on the } \\
\text { operated area }\end{array}$ & Craniotomy (surgery) \\
\hline $\begin{array}{l}\text { Balanites } \\
\text { aegyptiaca (L.) Del. }\end{array}$ & WBHG/13/011 & Tuyunwo & Desert date & Balanitaceae & Tree & $\begin{array}{l}\text { Lowland/ } \\
\text { escarpment }\end{array}$ & Leaves, fruits & Cooked as vegetable & Healing vegetable \\
\hline $\begin{array}{l}\text { Balanites rotundifolia } \\
\text { (Tiegh.) Blatt }\end{array}$ & WBHG/13/118 & Lomion & Not found & Balanitaceae & Tree & $\begin{array}{l}\text { Lowland/ } \\
\text { escarpment }\end{array}$ & Fruits & $\begin{array}{l}\text { Crush the fruits, soak } \\
\text { in water and apply }\end{array}$ & Pesticide \\
\hline $\begin{array}{l}\text { Berchemia discolor } \\
\text { (Klotsch) Hemsl }\end{array}$ & WBHG/13/076 & Muchukwo & Bird plum & Rhamnaceae & Tree & $\begin{array}{l}\text { Lowland/ } \\
\text { escarpment }\end{array}$ & Roots & Boiled and administered & Erectile dysfunction \\
\hline
\end{tabular}


Table 1 List of the various Herbal plants used by herbalistsand corresponding uses (Continued)

\begin{tabular}{|c|c|c|c|c|c|c|c|c|c|}
\hline Boscia coriacea Pax. & WBHG/13/062 & Sorukwo & Not found & Capparidaceae & Tree & $\begin{array}{l}\text { Lowland/ } \\
\text { escarpment }\end{array}$ & Seeds & Boiled and administered & Obesity \\
\hline Bryophyta spp. & WBHG/13/096 & Chebumbu & Green Moss & Oxallidaceae & Herb & $\begin{array}{l}\text { Highland/ } \\
\text { escarpment }\end{array}$ & Whole plant & Burnt and ash licked & $\begin{array}{l}\text { Heartburn/Teething in } \\
\text { children }\end{array}$ \\
\hline Bryophyta spp. & WBHG/13/096 & Chebumbu & Green Moss & Oxallidaceae & Herb & $\begin{array}{l}\text { Highland/ } \\
\text { escarpment }\end{array}$ & Whole plant & $\begin{array}{l}\text { Burnt and ashes } \\
\text { rubbed on the gums }\end{array}$ & $\begin{array}{l}\text { To relive pain associated } \\
\text { with teething in children }\end{array}$ \\
\hline $\begin{array}{l}\text { Calotropis procera } \\
\text { (Aiton) W.T. Aiton }\end{array}$ & WBHG/13/079 & Kibou & $\begin{array}{l}\text { Giant milkweed/ } \\
\text { calotropis }\end{array}$ & Asclepiadaceae & Shrub & Lowland & Leaves & $\begin{array}{l}\text { Burnt and ash } \\
\text { administered }\end{array}$ & Emetic \\
\hline $\begin{array}{l}\text { Capparis } \\
\text { cartilaginea Decne. }\end{array}$ & WBHG/13/091 & Chepteretwo & Caper shrub & Capparidaceae & Shrub & Lowland & Leaves & $\begin{array}{l}\text { Chewed/ burnt } \\
\text { and ash licked }\end{array}$ & Heartburn/peptic ulcers \\
\hline $\begin{array}{l}\text { Capparis } \\
\text { cartilaginea Decne. }\end{array}$ & WBHG/13/091 & Chepteretwo & Caper shrub & Capparidaceae & Shrub & Lowland & Flowers & $\begin{array}{l}\text { Dried, crushed and } \\
\text { added to meat }\end{array}$ & Food flavour \\
\hline $\begin{array}{l}\text { Caralluma } \\
\text { acutangula }\end{array}$ & WBHG/13/031 & Mochontopokot & Caralluma & Asclepiadaceae & Herb & Lowland & Whole plant & Whole plant chewed & $\begin{array}{l}\text { Chest congestion } \\
\text { (wheezing) }\end{array}$ \\
\hline $\begin{array}{l}\text { Carissa edulis } \\
\text { (Forssk.) Vahl }\end{array}$ & WBHG/13/091 & Legetetwet & Egyptian carissa & Apocynaceae & Shrub & $\begin{array}{l}\text { Highland/ } \\
\text { escarpment/ } \\
\text { lowland }\end{array}$ & Roots & $\begin{array}{l}\text { Boiled and added to } \\
\text { other herbs or used } \\
\text { alone }\end{array}$ & General malaise \\
\hline $\begin{array}{l}\text { Carissa edulis } \\
\text { (Forssk.) Vahl }\end{array}$ & WBHG/13/091 & Legetetwet & Egyptian carissa & Apocynaceae & Shrub & $\begin{array}{l}\text { Highland/ } \\
\text { escarpment/ } \\
\text { lowland }\end{array}$ & Fruits & Consumed in whole & Appetizer \\
\hline $\begin{array}{l}\text { Cassia } \\
\text { didymobotrya Fres. }\end{array}$ & WBHG/13/094 & Senetwo & Peanut- butter cassia & Caesalpiniaceae & Shrub & $\begin{array}{l}\text { Highland/ } \\
\text { escarpment }\end{array}$ & Dried bark & $\begin{array}{l}\text { Pound and applied } \\
\text { on bleeding area }\end{array}$ & $\begin{array}{l}\text { Antihaemorrhagic } \\
\text { (stops bleeding) }\end{array}$ \\
\hline $\begin{array}{l}\text { Cassia } \\
\text { didymobotrya Fres. }\end{array}$ & WBHG/13/094 & Senetwo & Peanut- butter cassia & Caesalpiniaceae & Shrub & $\begin{array}{l}\text { Highland/ } \\
\text { escarpment }\end{array}$ & Leaves/bark & Boiled and administered & Hypertension \\
\hline $\begin{array}{l}\text { Cassia obtusifolia } \\
\text { Linn or Cassia } \\
\text { occidentalisL. }\end{array}$ & $\begin{array}{l}\text { WBHG/13/070or } \\
\text { 070a }\end{array}$ & Kipkurkuris & Senna & Caesalpiniaceae & Herb & $\begin{array}{l}\text { Lowland/ } \\
\text { escarpment }\end{array}$ & $\begin{array}{l}\text { Leaves and } \\
\text { seeds }\end{array}$ & Boiled and administered & Abortifacient \\
\hline $\begin{array}{l}\text { Cirsium vulgare } \\
\text { (Savi.) Ten }\end{array}$ & WBHG/13/013 & Tokoukowo & Spear thistle & Asteraceae & Herb & $\begin{array}{l}\text { Highland/ } \\
\text { escarpment }\end{array}$ & Leaves/twigs & Burnt and ash licked & Heartburn \\
\hline $\begin{array}{l}\text { Cirsium vulgare } \\
\text { (Savi.) Ten }\end{array}$ & WBHG/13/013 & Tokoukowo & Spear Thistle & Asteraceae & Herb & $\begin{array}{l}\text { Highland/ } \\
\text { escarpment }\end{array}$ & Leaves/Twigs & $\begin{array}{l}\text { Burnt ash applied on } \\
\text { incisions made on } \\
\text { the skin }\end{array}$ & Talisman \\
\hline $\begin{array}{l}\text { Clerodendrum } \\
\text { myricoides } \\
\text { (Hochst.) Vatke }\end{array}$ & WBHG/13/005 & Chebobet & Blue glory bower & Lamiaceae & Tree & Escarpment & Leaves & Burnt and ash licked & "Kipei" condition \\
\hline $\begin{array}{l}\text { Clutia abyssinica } \\
\text { Jaub \& Spach }\end{array}$ & WBHG/13/089 & Kapkurelwo & $\begin{array}{l}\text { Smooth-fruited } \\
\text { lightning-bush }\end{array}$ & Euphorbiaceae & Shrub & $\begin{array}{l}\text { Lowland/ } \\
\text { riverine }\end{array}$ & Roots & $\begin{array}{l}\text { Boiled and mixed } \\
\text { with other herbs }\end{array}$ & Synergistic herb \\
\hline $\begin{array}{l}\text { Clutia abyssinica } \\
\text { Jaub \& Spach }\end{array}$ & WBHG/13/089 & Kapkurelwo & $\begin{array}{l}\text { Smooth-fruited } \\
\text { lightning-bush }\end{array}$ & Euphorbiaceae & Shrub & $\begin{array}{l}\text { Lowland/ } \\
\text { riverine }\end{array}$ & Roots & Boiled and administered & Erectile dysfunction \\
\hline $\begin{array}{l}\text { Coccinia grandis } \\
\text { (L.) Voigt }\end{array}$ & WBHG/13/019 & Minjilwo & Ivy ground & Cucurbitaceae & Herb & Lowland & Leaves & $\begin{array}{l}\text { Boiled and used as } \\
\text { vegetable }\end{array}$ & $\begin{array}{l}\text { Treatment of heavy } \\
\text { snoring }\end{array}$ \\
\hline
\end{tabular}


Table 1 List of the various Herbal plants used by herbalistsand corresponding uses (Continued)

\begin{tabular}{|c|c|c|c|c|c|c|c|c|c|}
\hline $\begin{array}{l}\text { Combretum } \\
\text { apiculatum Sond. }\end{array}$ & WBHG/13/111 & Leleiya & Red bushwillow & Combretaceae & Tree & Escarpment & Fruit bodies & Burnt and ash licked & Cancer \\
\hline $\begin{array}{l}\text { Commicarpus } \\
\text { africanus } \\
\text { (Lour.) Dandy }\end{array}$ & WBHG/13/065 & Tanagit & Comicarp (Catalan) & Nyctaginaceae & Herb & Escarpment & Leaves & $\begin{array}{l}\text { Crushed leaves rubbed } \\
\text { on the ganglion }\end{array}$ & Removal of ganglion \\
\hline Cordia sinensis Lam. & WBHG/13/007 & Adomeyon & $\begin{array}{l}\text { Grey-leaved saucer } \\
\text { berry }\end{array}$ & Boraginaceae & Tree & Lowland & Twigs & $\begin{array}{l}\text { Break tender twigs } \\
\text { for use }\end{array}$ & Toothbrush \\
\hline $\begin{array}{l}\text { Crassocephalum } \\
\text { mannii }\end{array}$ & WBHG/13/042 & Terkekwo & Thickhead & Asteraceae & Shrub & $\begin{array}{l}\text { Highland/ } \\
\text { escarpment }\end{array}$ & Roots & Boiled and administered & Arthritis \\
\hline Crotalaria incana L. & WBHG/13/060 & Kimira & Woolly Rattlepod & Fabaceae & Herb & $\begin{array}{l}\text { Lowland/ } \\
\text { escarpment }\end{array}$ & Leaves & $\begin{array}{l}\text { Boiled and used as } \\
\text { vegetable }\end{array}$ & Anaemia \\
\hline Crotalaria incana $\mathrm{L}$. & WBHG/13/060 & Kimira & Woolly Rattlepod & Fabaceae & Herb & $\begin{array}{l}\text { Lowland/ } \\
\text { escarpment }\end{array}$ & Leaves & Cooked as vegetable & Healing vegetable \\
\hline $\begin{array}{l}\text { Croton } \\
\text { ciliatoglandulifer } \\
\text { Ortega }\end{array}$ & WBHG/13/077 & Kibichan & Mexican croton & Euphorbiaceae & $\begin{array}{l}\text { Lianal } \\
\text { climber }\end{array}$ & $\begin{array}{l}\text { Lowland/ } \\
\text { escarpment }\end{array}$ & Roots & $\begin{array}{l}\text { Boiled and mixed } \\
\text { with other herbs }\end{array}$ & Synergistic herb \\
\hline $\begin{array}{l}\text { Croton } \\
\text { ciliatoglandulifer } \\
\text { Ortega }\end{array}$ & WBHG/13/077 & Kibichan & Mexican croton & Euphorbiaceae & $\begin{array}{l}\text { Lianal } \\
\text { climber }\end{array}$ & $\begin{array}{l}\text { Lowland/ } \\
\text { escarpment }\end{array}$ & Roots & $\begin{array}{l}\text { Chewed raw/ boiled } \\
\text { with other herbs }\end{array}$ & Heartburn \\
\hline $\begin{array}{l}\text { Croton } \\
\text { dichogamus Pax }\end{array}$ & WBHG/13/064 & Kerelwo & Orange leavedcroton & Euphorbiaceae & Shrub & Escarpment & $\begin{array}{l}\text { Higher parasites } \\
\text { on this plant }\end{array}$ & Burnt and ash licked & $\begin{array}{l}\text { "Kipei" condition } \\
\text { (abdominal pain and } \\
\text { oral thrush) }\end{array}$ \\
\hline $\begin{array}{l}\text { Croton } \\
\text { dichogamus Pax }\end{array}$ & WBHG/13/064 & Kerelwo & Orange leavedcroton & Euphorbiaceae & Shrub & Escarpment & $\begin{array}{l}\text { Bark, roots and } \\
\text { flowers }\end{array}$ & Boiled and administered & $\begin{array}{l}\text { Chest congestion } \\
\text { (wheezing) }\end{array}$ \\
\hline $\begin{array}{l}\text { Croton } \\
\text { megalocarpus } \\
\text { Hutch. }\end{array}$ & WBHG/13/045 & Otonwet & Broadleaved croton & Euphorbiaceae & Tree & Escarpment & Roots & Boiled and administered & Purgative \\
\hline $\begin{array}{l}\text { Cucumisdipsaceu s } \\
\text { C. G. Ehrenb. } \\
\text { ex Spac }\end{array}$ & WBHG/13/055 & Kisangwa & Bitter gourd & Cucurbitaceae & Herb & $\begin{array}{l}\text { Lowland/ } \\
\text { escarpment }\end{array}$ & Fruits & $\begin{array}{l}\text { Crushed and mixed } \\
\text { with little water and } \\
\text { administered to patient }\end{array}$ & Emetic \\
\hline Cyperus esculentus L. & WBHG/13/103 & Morgut & Yellow nutsedge & Cyperaceae & Herb & $\begin{array}{l}\text { Lowland/ } \\
\text { swampy areas }\end{array}$ & Tubers & Chewed & Stomachache \\
\hline Cyperus esculentus L. & WBHG/13/103 & Morgut & Yellow nutsedge & Cyperaceae & Herb & $\begin{array}{l}\text { Lowland/ } \\
\text { swampy areas }\end{array}$ & Tubers & Chewed & $\begin{array}{l}\text { Abdominal (colic) } \\
\text { pains/Talisman }\end{array}$ \\
\hline Cyperus esculentus L. & WBHG/13/103 & Morgut & Yellow nutsedge & Cyperaceae & Herb & $\begin{array}{l}\text { Lowland/ } \\
\text { swampy areas }\end{array}$ & Tubers & Chewed or boiled & Colic pain in children \\
\hline Cyperus esculentus L. & WBHG/13/103 & Morgut & Yellow nutsedge & Cyperaceae & Herb & $\begin{array}{l}\text { Lowland/ } \\
\text { swampy areas }\end{array}$ & Tubers & Chewed and applied & Talisman \\
\hline $\begin{array}{l}\text { Cyphostemma } \\
\text { serpens (Hochst. } \\
\text { exA.Rich.) Desc }\end{array}$ & WBHG/13/021 & Kirorot & Not found & Vitaceae & Herb & $\begin{array}{l}\text { Lowland/ } \\
\text { escarpment }\end{array}$ & Dried stem & Boiled and administered & Abortifacient \\
\hline
\end{tabular}


Table 1 List of the various Herbal plants used by herbalistsand corresponding uses (Continued)

\begin{tabular}{|c|c|c|c|c|c|c|c|c|c|}
\hline $\begin{array}{l}\text { Cyphostemma } \\
\text { cyphopetalum } \\
\text { (Fresen.) Descoings }\end{array}$ & WBHG/13/075 & Kibungwach & Not found & Vitaceae & Herb & Escarpment & Tubers & $\begin{array}{l}\text { Crush and apply on } \\
\text { the pests }\end{array}$ & Pesticide \\
\hline $\begin{array}{l}\text { Dactylocteniuma } \\
\text { egyptium L. }\end{array}$ & WBHG/13/073 & Anyinya & $\begin{array}{l}\text { Egyptian crowfoot } \\
\text { grass }\end{array}$ & Poaceae & Herb & $\begin{array}{l}\text { Lowland/ } \\
\text { escarpment }\end{array}$ & Leaves & Crushed and chewed raw & Heartburn \\
\hline $\begin{array}{l}\text { Dactylocteniuma } \\
\text { egyptium L. }\end{array}$ & WBHG/23/073 & Anyinya & $\begin{array}{l}\text { Egyptian crowfoot } \\
\text { grass }\end{array}$ & Poaceae & Herb & $\begin{array}{l}\text { Lowland/ } \\
\text { escarpment }\end{array}$ & Whole plant & Cooked as vegetable & Healing vegetable \\
\hline Digeramuricata L. & WBHG/13/099 & Chesugut & False Amaranth & Amaranthaceae & Herb & Lowland & Leaves & Cooked as vegetable & Healing vegetable \\
\hline $\begin{array}{l}\text { Diospyros scabra } \\
\text { (Chiov.) Cufod }\end{array}$ & WBHG/13/016 & Turetwo & Ebony tree & Ebenaceae & Tree & Escarpment & Bark & $\begin{array}{l}\text { Boil and patient } \\
\text { bathed in concoction } \\
\text { and covered }\end{array}$ & Fever in children \\
\hline $\begin{array}{l}\text { Diospyros scabra } \\
\text { (Chiov.) Cufod }\end{array}$ & WBHG/13/016 & Turetwo & Ebony tree & Ebenaceae & Tree & Escarpment & Twigs & $\begin{array}{l}\text { Break tender twigs } \\
\text { for use }\end{array}$ & Toothbrush \\
\hline $\begin{array}{l}\text { Dodonaea viscose } \\
\text { (L.) Jacq. }\end{array}$ & WBHG/13/098 & Tebelekwo & Hopbush & Sapindaceae & Shrub & Escarpment & Twigs & $\begin{array}{l}\text { Break tender twigs } \\
\text { and use }\end{array}$ & Toothbrush \\
\hline Dolichos spp. & WBHG/13/104 & Kipchoror & Hyacinth bean & Fabaceae & Herb & Lowland & Dry leaves & $\begin{array}{l}\text { Pound, mixed with } \\
\text { water and administered }\end{array}$ & $\begin{array}{l}\text { Enhance lactation in } \\
\text { breastfeeding mothers }\end{array}$ \\
\hline $\begin{array}{l}\text { Drimia indica } \\
\text { (Roxb.) Jessop }\end{array}$ & WBHG/13/046 & Barangoya & Indian-squill & Asparagaceae & Herb & $\begin{array}{l}\text { Lowland/ } \\
\text { escarpment }\end{array}$ & Bulbs & Bulb applied on the ulcers & Cancer \\
\hline $\begin{array}{l}\text { Ehretia cymose } \\
\text { var. silvatica Guerke }\end{array}$ & WBHG/13/066 & $\begin{array}{l}\text { Kabonbonet or } \\
\text { Mororion }\end{array}$ & Stamperwood & Boraginaceae & Shrub & Highland & Bark & Boiled and administered & Stomachache \\
\hline $\begin{array}{l}\text { Ekebergia } \\
\text { rueppelliana Fres }\end{array}$ & WBHG/13/045 & Kerbu & Ekebergia & Meliaceae & Tree & Lowland & Bark & Boiled and administered & Purgative \\
\hline $\begin{array}{l}\text { Ensete ventricosum } \\
\text { (Welw.) E.E. Cheesman }\end{array}$ & WBHG/13/002 & Sosurwo & False banana & Musaceae & Shrub & $\begin{array}{l}\text { Highland/ } \\
\text { escarpment }\end{array}$ & Tip end (flower) & $\begin{array}{l}\text { Crushed, dried and } \\
\text { burnt. Ash licked }\end{array}$ & Heartburn \\
\hline $\begin{array}{l}\text { Erythrina } \\
\text { abyssinica DC. }\end{array}$ & WBHG/13/029 & Korkorwo & Corpse flower & Fabaceae & Tree & Highland & Bark & Boiled and administered & Mumps \\
\hline $\begin{array}{l}\text { Euclea divinorum } \\
\text { Hiern. }\end{array}$ & WBHG/13/052 & Uswo & Magic Guarri & Ebenaceae & Shrub & Escarpment & Twigs & Break tender twigs for use & Toothbrush \\
\hline EucleadivinorumHiern. & WBHG/13/052 & Uswo & Magic Guarri & Ebenaceae & Shrub & Escarpment & Bark & $\begin{array}{l}\text { Pound and applied } \\
\text { on the incision made } \\
\text { on the bitten site }\end{array}$ & Antivenom \\
\hline $\begin{array}{l}\text { Faidherbia albida } \\
\text { (Delile) A.Chev. }\end{array}$ & WBHG/13/027 & Kokocha & Apple ring acacia & Mimosaceae & Tree & $\begin{array}{l}\text { Lowland/ } \\
\text { riverine/ } \\
\text { swampy areas }\end{array}$ & Fruit bodies & Burnt and ash licked & Cancer \\
\hline $\begin{array}{l}\text { Faurea saligna } \\
\text { Harvey }\end{array}$ & WBHG/13/018 & Sirirte & Beechwood & Proteaceae & Tree & Highland & Bark & Boiled and administered & Period pains \\
\hline $\begin{array}{l}\text { Flacourtia indica } \\
\text { (Burm.f.) Merr. }\end{array}$ & WBHG/13/072 & Tingoswo & Governor's plum & Flacourtiaceae & Tree & $\begin{array}{l}\text { Lowland/ } \\
\text { escarpment }\end{array}$ & Fruits & Dry the fruit and use & Fermentation \\
\hline $\begin{array}{l}\text { Fuerstiaafricana } \\
\text { T.C.E. Fries }\end{array}$ & WBHG/13/004 & Kipirirwo & Not found & Lamiaceae & Herb & Escarpment & Leaves & Crushed and sap instilled & Eye ailments \\
\hline
\end{tabular}


Table 1 List of the various Herbal plants used by herbalistsand corresponding uses (Continued)

\begin{tabular}{|c|c|c|c|c|c|c|c|c|c|}
\hline $\begin{array}{l}\text { Fuerstia africana } \\
\text { T.C.E. Fries }\end{array}$ & WBHG/13/004 & Kipirirwo & Not found & Lamiaceae & Herb & Escarpment & Leaves & $\begin{array}{l}\text { Crushed and sap } \\
\text { applied }\end{array}$ & Toothache \\
\hline Gardenia sp & WBHG/13/044 & $\begin{array}{l}\text { Mogilion/ } \\
\text { Kobilwo }\end{array}$ & Forest gardenia & Rubiaceae & Tree & Lowland & Fruits & $\begin{array}{l}\text { Crushed and } \\
\text { administered to patient }\end{array}$ & Emetic/purgative \\
\hline Grewia villosa Willd & WBHG/13/020 & Mokurwo & Mallow raisin & Tiliaceae & Shrub & Lowland & Leaves & $\begin{array}{l}\text { Cover milk gourd } \\
\text { with it }\end{array}$ & Fermentation \\
\hline $\begin{array}{l}\text { Gynandropsis } \\
\text { gynandra (L.) Briq. }\end{array}$ & WBHG/13/025 & Sachan & African spider flower & Capparidaceae & Herb & $\begin{array}{l}\text { Lowland/ } \\
\text { escarpment }\end{array}$ & Leaves & $\begin{array}{l}\text { Cooked and used as } \\
\text { vegetable }\end{array}$ & Healing vegetable \\
\hline $\begin{array}{l}\text { Heeria reticulata } \\
\text { (Baker f.) Engl }\end{array}$ & WBHG/13/008 & Mutung'wo & Raisin bush & Anacardiaceae & Tree & Escarpment & Bark & Burnt and ash licked & $\begin{array}{l}\text { Removal of retained } \\
\text { placenta in both } \\
\text { human and animals }\end{array}$ \\
\hline $\begin{array}{l}\text { Heeria reticulata } \\
\text { (Baker f.) Engl }\end{array}$ & WBHG/13/008 & Mutung'wo & Raisin bush & Anacardiaceae & Tree & Escarpment & Leaves & $\begin{array}{l}\text { Crush, soak in water } \\
\text { and apply }\end{array}$ & Constipation in animals \\
\hline $\begin{array}{l}\text { Hos/undia } \\
\text { opposite Valh. }\end{array}$ & WBHG/13/083 & Sumeyon & Orange bird berry & Lamiaceae & Shrub & $\begin{array}{l}\text { Lowland/ } \\
\text { escarpment }\end{array}$ & Leaves, roots & Boiled and administered & Colic pain in children \\
\hline $\begin{array}{l}\text { Hoslundia } \\
\text { opposite Valh. }\end{array}$ & WBHG/13/083 & Sumeyon & Orange bird berry & Lamiaceae & Shrub & $\begin{array}{l}\text { Lowland/ } \\
\text { escarpment }\end{array}$ & Roots, leaves & Boiled and administered & Amoebiasis \\
\hline $\begin{array}{l}\text { Hos/undia } \\
\text { opposite Valh. }\end{array}$ & WBHG/13/083 & Sumeyon & Orange bird berry & Lamiaceae & Shrub & $\begin{array}{l}\text { Lowland/ } \\
\text { escarpment }\end{array}$ & Leaves & $\begin{array}{l}\text { Crushed, soaked } \\
\text { in water and gargled }\end{array}$ & Oral thrush \\
\hline $\begin{array}{l}\text { Hypoestes forskaolii } \\
\text { (Vahl) Roem. \& } \\
\text { Schult. }\end{array}$ & WBHG/13/059 & $\begin{array}{l}\text { Sirkonwol } \\
\text { Kaberkewo }\end{array}$ & White ribbon bush & Acanthaceae & Shrub & $\begin{array}{l}\text { Lowland/ } \\
\text { escarpment }\end{array}$ & Leaves, twigs & $\begin{array}{l}\text { Crush, soak in water } \\
\text { and apply }\end{array}$ & Pesticide \\
\hline Iboza spp. & WBHG/13/012 & Lonwo & Nutmeg bush & Lamiaceae & Shrub & Escarpment & leaves & Crushed and pound & Perfume \\
\hline Indigofera arrecta L. & WBHG/13/034 & Sargellat & African indigo & Fabaceae & Herb & $\begin{array}{l}\text { Lowland/ } \\
\text { escarpment }\end{array}$ & Roots & $\begin{array}{l}\text { Boiled and mixed } \\
\text { with other herbs }\end{array}$ & Synergistic herb \\
\hline Indigofera arrecta L & WBHG/13/034 & Sargellat & African indigo & Fabaceae & Herb & $\begin{array}{l}\text { Lowland/ } \\
\text { escarpment }\end{array}$ & Roots & Boiled and administered & Stomachache \\
\hline Indigofera arrecta L & WBHG/13/034 & Sargellat & African indigo & Fabaceae & Herb & $\begin{array}{l}\text { Lowland/ } \\
\text { escarpment }\end{array}$ & Roots & $\begin{array}{l}\text { Boiled \& mixed with } \\
\text { other herbs }\end{array}$ & Cancer \\
\hline Indigofera arrecta L & WBHG/13/034 & Sargellat & African indigo & Fabaceae & Herb & $\begin{array}{l}\text { Lowland/ } \\
\text { escarpment }\end{array}$ & Roots & Chewed & Toothache \\
\hline $\begin{array}{l}\text { Ipomoea } \\
\text { lapidosavatke }\end{array}$ & WBHG/13/078 & Kimugugu & $\begin{array}{l}\text { Common morning } \\
\text { glory }\end{array}$ & Convolvulaceae & $\begin{array}{l}\text { Lianal } \\
\text { climber }\end{array}$ & $\begin{array}{l}\text { Lowland/ } \\
\text { escarpment }\end{array}$ & Stem & Boiled and administered & $\begin{array}{l}\text { Sexually transmitted } \\
\text { diseases }\end{array}$ \\
\hline Justica spp. & WBHG/13/024 & Kepkalomion & Jacobinia & Acanthaceae & Herb & Escarpment & Leaves & Boiled and administered & Abdominal (colic) pains \\
\hline $\begin{array}{l}\text { Kalanchoe } \\
\text { germanae } \\
\text { Raym.-Hamet ex } \\
\text { Raadts }\end{array}$ & WBHG/13/030 & Kibarbany & Air plant & Crassulaceae & Herb & $\begin{array}{l}\text { Lowland/ } \\
\text { escarpment }\end{array}$ & Leaves & $\begin{array}{l}\text { Pound and rubbed on } \\
\text { ganglion area }\end{array}$ & Removal of ganglion \\
\hline $\begin{array}{l}\text { Kalanchoe } \\
\text { germanae Raym.- } \\
\text { Hamet ex Raadts }\end{array}$ & WBHG/13/030 & Kibarbany & Air plant & Crassulaceae & Herb & $\begin{array}{l}\text { Lowland/ } \\
\text { escarpment }\end{array}$ & Leaves & $\begin{array}{l}\text { Pound and rubbed on } \\
\text { painful area }\end{array}$ & Poultice \\
\hline
\end{tabular}


Table 1 List of the various Herbal plants used by herbalistsand corresponding uses (Continued)

\begin{tabular}{|c|c|c|c|c|c|c|c|c|c|}
\hline $\begin{array}{l}\text { Kigelia Africana } \\
\text { (Lam.) Beneth }\end{array}$ & WBHG/13/048 & Rotion & African sausage & Bignoniaceae & Tree & $\begin{array}{l}\text { Lowland/ } \\
\text { escarpment }\end{array}$ & Fruits & Split and dry & $\begin{array}{l}\text { For fermentation } \\
\text { during the brewing of } \\
\text { traditional beer }\end{array}$ \\
\hline $\begin{array}{l}\text { Lannea fulva } \\
\text { (Engl.) Engl. }\end{array}$ & WBHG/13/056 & Lolotwo & Not found & Anacardiaceae & Tree & $\begin{array}{l}\text { Lowland/ } \\
\text { escarpment }\end{array}$ & Bark & $\begin{array}{l}\text { Pound and mixed } \\
\text { with other herbs }\end{array}$ & Antivenom \\
\hline Lantana trifolia L. & WBHG/13/046 & Bekaptarit & $\begin{array}{l}\text { Three- } \\
\text { leafshrubverbena }\end{array}$ & Verbenaceae & Shrub & $\begin{array}{l}\text { Lowland/ } \\
\text { escarpment }\end{array}$ & Leaves/twigs & Boiled and administered & Abdominal (colic) pains \\
\hline Lantana trifolia L. & WBHG/13/046 & Bekaptarit & $\begin{array}{l}\text { Three- } \\
\text { leafshrubverbena }\end{array}$ & Verbenaceae & Shrub & $\begin{array}{l}\text { Lowland/ } \\
\text { escarpment }\end{array}$ & $\begin{array}{l}\text { Leaves, twigs \& } \\
\text { fruits }\end{array}$ & Boiled and administered & $\begin{array}{l}\text { Enhance lactation in } \\
\text { breastfeeding mothers }\end{array}$ \\
\hline $\begin{array}{l}\text { Leptadenia hastate } \\
\text { (Pers.) Decne. }\end{array}$ & WBHG/13/058 & Kipchegin & Not found & Apocynaceae & $\begin{array}{l}\text { Lianal } \\
\text { climber }\end{array}$ & $\begin{array}{l}\text { Lowland/ } \\
\text { escarpment }\end{array}$ & Tender leaves & Cooked as vegetable & Healing vegetable \\
\hline $\begin{array}{l}\text { Lippia javanica } \\
\text { (Burm. f.) Spreng }\end{array}$ & WBHG/13/053 & Chebokobil & Zinziba plant & Verbenaceae & Shrub & Highland & $\begin{array}{l}\text { Leaves and } \\
\text { twigs }\end{array}$ & Boiled and administered & Amoebiasis \\
\hline $\begin{array}{l}\text { Lippia javanica } \\
\text { (Burm. f.) Spreng }\end{array}$ & WBHG/13/053 & Chebokobil & Zinziba plant & Verbenaceae & Shrub & Highland & $\begin{array}{l}\text { Leaves and } \\
\text { twigs }\end{array}$ & $\begin{array}{l}\text { Boiled together with } \\
\text { maize, cassava, } \\
\text { groundnuts and } \\
\text { even tea }\end{array}$ & Food flavour \\
\hline $\begin{array}{l}\text { Maesa lanceolata } \\
\text { Forsk. }\end{array}$ & WBHG/13/087 & Mborion & False assegai & Myrsinaceae & $\begin{array}{l}\text { Lianal } \\
\text { climber }\end{array}$ & Lowland & Whole plant & $\begin{array}{l}\text { Crush, soak in water } \\
\text { and apply }\end{array}$ & $\begin{array}{l}\text { Veterinary use as } \\
\text { antipoison }\end{array}$ \\
\hline Nuxia congesta Fres & WBHG/13/082 & Chorwa & Brittle wood & Loganiaceae & Tree & Highland & Roots & Boiled and administered & $\begin{array}{l}\text { Abdominal (colic) } \\
\text { pains /Flu }\end{array}$ \\
\hline Nuxia congesta Fres & WBHG/13/082 & Chorwa & Brittle wood & Loganiaceae & Tree & Highland & Roots & Boiled and administered & Flu \\
\hline Ocimum basilicum L. & WBHG/13/028 & Klachir & Basil & Lamiaceae & Herb & Lowland & Leaves & $\begin{array}{l}\text { Crushed and sap instilled } \\
\text { on the affected eye }\end{array}$ & Eye ailments \\
\hline Olea Africana Mill. & WBHG/13/032 & Remit & Wild olive & Oleaceae & Tree & $\begin{array}{l}\text { Highland/ } \\
\text { escarpment }\end{array}$ & Dried bark & $\begin{array}{l}\text { Pound and powder } \\
\text { applied }\end{array}$ & Eye ailments \\
\hline Olea Africana Mill. & WBHG/13/032 & Remit & Wild olive & Oleaceae & Tree & $\begin{array}{l}\text { Highland/ } \\
\text { escarpment }\end{array}$ & Bark & Boiled and administered & Itchy rashes \\
\hline $\begin{array}{l}\text { Ornithogalum } \\
\text { tenuifolium } \\
\text { Delaroche }\end{array}$ & WBHG/13/050 & Katagwa & Sea-onion & Hyacinthaceae & Herb & $\begin{array}{l}\text { Lowland/ } \\
\text { escarpment }\end{array}$ & Tubers & $\begin{array}{l}\text { Crushed and applied } \\
\text { on joints }\end{array}$ & Arthritis \\
\hline $\begin{array}{l}\text { Pappea capensis } \\
\text { Eckyl \& Zeyh }\end{array}$ & WBHG/13/023 & Kibiryokwo & Jacket plum & Sapindaceae & Tree & $\begin{array}{l}\text { Lowland/ } \\
\text { escarpment }\end{array}$ & Fruit bodies & Burnt and ash licked & Cancer \\
\hline $\begin{array}{l}\text { Pappea capensis } \\
\text { Eckyl \& Zeyh }\end{array}$ & WBHG/13/023 & Kibiryokwo & Jacket plum & Sapindaceae & Tree & $\begin{array}{l}\text { Lowland/ } \\
\text { escarpment }\end{array}$ & Higher parasites & Burnt and ashes licked & Rheumatism \\
\hline $\begin{array}{l}\text { Pavetta abyssinica } \\
\text { Fresen }\end{array}$ & WBHG/13/107 & Cheptabirbirwo & Not found & Rubiaceae & Shrub & $\begin{array}{l}\text { Lowland/ } \\
\text { escarpment }\end{array}$ & Bark & Crushed and administered & Purgative \\
\hline $\begin{array}{l}\text { Pentas longiflora } \\
\text { W.R.B. Oliv. }\end{array}$ & WBHG/13/093 & Chebirbirgorok & Not found & Rubiaceae & Shrub & Escarpment & Fruits/bark & Boiled and administered & Malaria like symptoms \\
\hline $\begin{array}{l}\text { Periploca linearifolia } \\
\text { Dill. \& Rich. }\end{array}$ & WBHG/13/073 & Sinende & Not found & Apocynaceae & $\begin{array}{l}\text { Lianal } \\
\text { climber }\end{array}$ & Highland & Fruits/Leaves & & Ceremonial plant \\
\hline
\end{tabular}


Table 1 List of the various Herbal plants used by herbalistsand corresponding uses (Continued)

\begin{tabular}{|c|c|c|c|c|c|c|c|c|c|}
\hline $\begin{array}{l}\text { Periploca linearifolia } \\
\text { Dill. \& Rich. }\end{array}$ & WBHG/13/073 & Sinende & Not found & Apocynaceae & $\begin{array}{l}\text { Lianal } \\
\text { climber }\end{array}$ & Highland & Fruits/Leaves & $\begin{array}{l}\text { Crush the fruits, soak } \\
\text { in water and apply }\end{array}$ & Pesticide \\
\hline $\begin{array}{l}\text { Podocarpus graciliar } \\
\text { Pilger }\end{array}$ & WBHG/13/067 & Bennet & $\begin{array}{l}\text { African fern treel } \\
\text { bastard yellow wood }\end{array}$ & Podocarpaceae & Tree & Highland & Bark & Boiled and administered & Hypertension \\
\hline $\begin{array}{l}\text { Portulaca } \\
\text { quadrifida L. }\end{array}$ & WBHG/13/037 & Kitumerio & $\begin{array}{l}\text { Small-leaved } \\
\text { purslane }\end{array}$ & Portulacaceae & Herb & Lowland & Whole plant & Cooked as vegetable & Healing vegetable \\
\hline Portulaca oleracea L. & WBHG/13/092 & Chemorin & Asthma weed & Portulacaceae & Herb & Lowland & $\begin{array}{l}\text { Whole crushed } \\
\text { plant }\end{array}$ & Boiled with other herbs & Cancer \\
\hline $\begin{array}{l}\text { Prunus africana } \\
\text { (Hook f.) Kalkm. }\end{array}$ & WBHG/13/014 & Tendwo & African plum tree & Rosaceae & Tree & Highland & Bark & Boiled and administered & Hypertension \\
\hline $\begin{array}{l}\text { Prunus Africana } \\
\text { (Hook f.) Kalkm. }\end{array}$ & WBHG/13/014 & Tendwo & African plum tree & Rosaceae & Tree & Highland & Bark/roots & Boiled and administered & Enlarged prostate \\
\hline $\begin{array}{l}\text { Psiadia arabica } \\
\text { Jaub.et Spach }\end{array}$ & WBHG/13/047 & Konocho & Not found & Asteraceae & Shrub & Escarpment & Bark & Boiled with water & Herbal "tea" \\
\hline Ricinus communis L. & WBHG/13/017 & Menwa & Castor bean & Euphorbiaceae & Shrub & $\begin{array}{l}\text { Lowland/ } \\
\text { escarpment }\end{array}$ & Seeds & Crushed and oil applied & $\begin{array}{l}\text { Treatment of hides } \\
\text { and skins }\end{array}$ \\
\hline Rumex acetosella L. & WBHG/13/080 & Kibongbong & Sheep sorrel & Polygonaceae & Shrub & $\begin{array}{l}\text { Highland/ } \\
\text { escarpment }\end{array}$ & Tubers & $\begin{array}{l}\text { Chewed or boiled and } \\
\text { administered }\end{array}$ & Hypertension \\
\hline Rumex acetosella $L$ & WBHG/13/080 & Kibongbong & Sheep sorrel & Polygonaceae & Shrub & $\begin{array}{l}\text { Highland/ } \\
\text { escarpment }\end{array}$ & Tubers & $\begin{array}{l}\text { Chewed or boiled } \\
\text { and administered }\end{array}$ & Diabetes \\
\hline $\begin{array}{l}\text { Saba comorensis } \\
\text { (Bojer ex A.DC.) } \\
\text { Pichon }\end{array}$ & WBHG/13/112 & Ochon & Rubber vine & Apocynaceae & $\begin{array}{l}\text { Lianal } \\
\text { climber }\end{array}$ & $\begin{array}{l}\text { Lowland/ } \\
\text { escarpment } \\
\text { (riverine) }\end{array}$ & Fruits & Fruit consumed & $\begin{array}{l}\text { Diagnosis of enlarged } \\
\text { prostate }\end{array}$ \\
\hline $\begin{array}{l}\text { Salvadora } \\
\text { persica Wall. }\end{array}$ & WBHG/13/033 & Chekowo & Toothbrush tree & Salvadoraceae & Tree & $\begin{array}{l}\text { Lowland/ } \\
\text { escarpment }\end{array}$ & Twigs & $\begin{array}{l}\text { Break tender twigs } \\
\text { and use }\end{array}$ & Toothbrush \\
\hline $\begin{array}{l}\text { Sansevieria } \\
\text { intermedia }\end{array}$ & WBHG/13/009 & Sorogat & $\begin{array}{l}\text { Mother-in-law } \\
\text { tongue }\end{array}$ & Agavaceae & Herb & $\begin{array}{l}\text { Lowland/ } \\
\text { escarpment }\end{array}$ & Leaves & Sap used as bait & Used to kill snakes \\
\hline $\begin{array}{l}\text { Schefflera } \\
\text { volkensii (Engl.) } \\
\text { Harms }\end{array}$ & WBHG/13/088 & Tingwon & Schefflera & Araliaceae & Tree & Highland & Dried resin & Resin sniffed & $\begin{array}{l}\text { Inhaled to clear } \\
\text { blocked nose }\end{array}$ \\
\hline $\begin{array}{l}\text { Schefflera volkensii } \\
\text { (Engl.) Harms }\end{array}$ & WBHG/13/088 & Tingwon & Schefflera & Araliaceae & Tree & Highland & Dried resin & Smear on body & Perfume \\
\hline $\begin{array}{l}\text { Sclerocarya birrea } \\
\text { (A. Rich.) Hochst }\end{array}$ & WBHG/13/106 & Orolwo & Amarula tree & Anacardiaceae & Tree & $\begin{array}{l}\text { Lowland/ } \\
\text { escarpment }\end{array}$ & Bark & $\begin{array}{l}\text { Chewed or boiled } \\
\text { and administered }\end{array}$ & Diabetes \\
\hline Solanum incanum L. & WBHG/13/035 & Kalobotwo & Thorn Apple & Solanaceae & Shrub & $\begin{array}{l}\text { Lowland/ } \\
\text { escarpment }\end{array}$ & Roots & Boiled or chewed raw & Abdominal (colic) pains \\
\hline Solanum incanum L. & WBHG/13/035 & Kalobotwo & Thorn Apple & Solanaceae & Shrub & $\begin{array}{l}\text { Lowland/ } \\
\text { escarpment }\end{array}$ & Roots & Boiled or chewed & Colic pain in children \\
\hline $\begin{array}{l}\text { Sphaeranthus } \\
\text { ukambensis } \\
\text { Vatke \& O.Hoffm }\end{array}$ & WBHG/13/085 & Moyon & Not found & Asteraceae & Shrub & Lowland & Roots & $\begin{array}{l}\text { Chewed or boiled and } \\
\text { administered }\end{array}$ & $\begin{array}{l}\text { Stomachache resulting } \\
\text { food poisoning }\end{array}$ \\
\hline
\end{tabular}


Table 1 List of the various Herbal plants used by herbalistsand corresponding uses (Continued)

\begin{tabular}{|c|c|c|c|c|c|c|c|c|c|}
\hline $\begin{array}{l}\text { Spilanthes } \\
\text { mauritiana } \\
\text { (Pers.) D.C }\end{array}$ & WBHG/13/090 & Kibutkut & Not found & Asteraceae & Herb & $\begin{array}{l}\text { Highland/ } \\
\text { escarpment/ } \\
\text { lowland }\end{array}$ & Whole plant & $\begin{array}{l}\text { Crushed and applied } \\
\text { on the affected tooth }\end{array}$ & Toothache \\
\hline $\begin{array}{l}\text { Spilanthes } \\
\text { mauritiana } \\
\text { (Pers.) D.C }\end{array}$ & WBHG/13/090 & Kibutkut & Not found & Asteraceae & Herb & $\begin{array}{l}\text { Highland/ } \\
\text { escarpment/ } \\
\text { lowland }\end{array}$ & $\begin{array}{l}\text { Whole crushed } \\
\text { plant }\end{array}$ & $\begin{array}{l}\text { Crushed and used as } \\
\text { mouthwash }\end{array}$ & Oral thrush \\
\hline $\begin{array}{l}\text { Spilanthes } \\
\text { mauritiana } \\
\text { (Pers.) D.C }\end{array}$ & WBHG/13/090 & Kibutkut & Not found & Asteraceae & Herb & $\begin{array}{l}\text { Highland/ } \\
\text { escarpment/ } \\
\text { lowland }\end{array}$ & Whole plant & $\begin{array}{l}\text { Crushed and applied } \\
\text { on the area to be } \\
\text { operated before } \\
\text { surgery }\end{array}$ & Craniotomy (surgery) \\
\hline $\begin{array}{l}\text { Sterculia Africana } \\
\text { (Lou.r) Fiori }\end{array}$ & WBHG/13/086 & llilwo & $\begin{array}{l}\text { African star } \\
\text { chestnut tree }\end{array}$ & Sterculiaceae & Tree & $\begin{array}{l}\text { Lowland/ } \\
\text { escarpment }\end{array}$ & Seeds & Chewed & Erectile dysfunction \\
\hline $\begin{array}{l}\text { Terminalia brownie } \\
\text { Fresen }\end{array}$ & WBHG/13/069 & Koloswo & Mbarao (in Swahili) & Combretaceae & Tree & Escarpment & Bark & Boiled or chewed raw & Abdominal (colic) pains \\
\hline $\begin{array}{l}\text { Terminalia brownie } \\
\text { Fresen }\end{array}$ & WBHG/13/069 & Koloswo & Mbarao (in Swahili) & Combretaceae & Tree & Escarpment & Bark & $\begin{array}{l}\text { Chewed or boiled } \\
\text { and administered }\end{array}$ & Jaundice \\
\hline $\begin{array}{l}\text { Terminalia } \\
\text { spinosa Engl. }\end{array}$ & WBHG/13/092 & Kitong'wo & Spiny cluster-leaf & Combretaceae & Tree & $\begin{array}{l}\text { Lowland/ } \\
\text { escarpment }\end{array}$ & Bark & Boiled and administered & Malaria like symptoms \\
\hline Tragiabrevipes Pax. & WBHG/13/069 & Kimelei & Shortspikenoseburn & Euphorbiaceae & $\begin{array}{l}\text { Lianal } \\
\text { climber }\end{array}$ & $\begin{array}{l}\text { Lowland/ } \\
\text { escarpment }\end{array}$ & Roots & $\begin{array}{l}\text { Pound and mixed } \\
\text { with other herbs }\end{array}$ & Antivenom \\
\hline Tribulusterrestris L. & WBHG/13/001 & Kilesan & Bullhead & Zygophylaceae & Herb & Lowland & $\begin{array}{l}\text { Whole crushed } \\
\text { plant }\end{array}$ & $\begin{array}{l}\text { Chewed or boiled and } \\
\text { administered }\end{array}$ & Erectile dysfunction \\
\hline Tribulusterrestris L. & WBHG/13/001 & Kilesan & Bullhead & Zygophylaceae & Herb & Lowland & Whole plant & Cooked as vegetable & Healing vegetable \\
\hline $\begin{array}{l}\text { Uvariasp var. } \\
\text { scheffleri }\end{array}$ & WBHG/13/010 & Murkuiyo & Not found & Annonaceae & $\begin{array}{l}\text { Shrub/ } \\
\text { climber }\end{array}$ & $\begin{array}{l}\text { Lowland/ } \\
\text { escarpment }\end{array}$ & Roots & Boiled and administered & Common colds/cough \\
\hline $\begin{array}{l}\text { Vangueria apiculata } \\
\text { K. Schum. }\end{array}$ & WBHG/13/003 & $\begin{array}{l}\text { Tabirirwo } \\
\text { (Komolwo } \\
\text { ne mining) }\end{array}$ & $\begin{array}{l}\text { Triangle-flowered } \\
\text { Wild-medlar }\end{array}$ & Rubiaceae & Shrub & $\begin{array}{l}\text { Highland/ } \\
\text { escarpment }\end{array}$ & Fruits & $\begin{array}{l}\text { Cooked as porridge and } \\
\text { consumed }\end{array}$ & Food supplement \\
\hline $\begin{array}{l}\text { Vangueria } \\
\text { madagascariensis } \\
\text { Gmel. }\end{array}$ & WBHG/13/041 & Komolwo neo & $\begin{array}{l}\text { Common wild } \\
\text { medlar }\end{array}$ & Rubiaceae & Shrub & $\begin{array}{l}\text { Highland/ } \\
\text { escarpment/ } \\
\text { lowland }\end{array}$ & Fruits & $\begin{array}{l}\text { Cooked as porridge } \\
\text { and consumed }\end{array}$ & Food supplement \\
\hline $\begin{array}{l}\text { Vernoniabrachycalyx } \\
\text { O. Hoffm. }\end{array}$ & WBHG/13/074 & $\begin{array}{l}\text { Kimagoi or } \\
\text { Chebongony }\end{array}$ & Ironweed & Asteraceae & Herb & Lowland & Roots & $\begin{array}{l}\text { Boiled and decoction } \\
\text { administered }\end{array}$ & Emetic \\
\hline $\begin{array}{l}\text { Warburgia ugandensis } \\
\text { Sprague }\end{array}$ & WBHG/13/109 & Sokwon & $\begin{array}{l}\text { East African green } \\
\text { wood }\end{array}$ & Canellaceae & Tree & $\begin{array}{l}\text { Highland/ } \\
\text { escarpment }\end{array}$ & Leaves & $\begin{array}{l}\text { Chewed or soaked } \\
\text { in hot water and } \\
\text { administered }\end{array}$ & Stomachache \\
\hline $\begin{array}{l}\text { Warburgia } \\
\text { ugandensis Sprague }\end{array}$ & WBHG/13/109 & Sokwon & $\begin{array}{l}\text { East African green } \\
\text { wood }\end{array}$ & Canellaceae & Tree & $\begin{array}{l}\text { Highland/ } \\
\text { escarpment }\end{array}$ & Bark & $\begin{array}{l}\text { Burnt and smoked } \\
\text { sniffed }\end{array}$ & Headache \\
\hline $\begin{array}{l}\text { Warburgia } \\
\text { ugandensis Sprague }\end{array}$ & WBHG/13/109 & Sokwon & $\begin{array}{l}\text { East African green } \\
\text { wood }\end{array}$ & Canellaceae & Tree & $\begin{array}{l}\text { Highland/ } \\
\text { escarpment }\end{array}$ & Leaves & Crush and applied & Toothache \\
\hline $\begin{array}{l}\text { Warburgia ugandensis } \\
\text { Sprague }\end{array}$ & WBHG/13/109 & Sokwon & $\begin{array}{l}\text { East African green } \\
\text { wood }\end{array}$ & Canellaceae & Tree & $\begin{array}{l}\text { Highland/ } \\
\text { escarpment }\end{array}$ & Leaves & $\begin{array}{l}\text { Chewed or soaked } \\
\text { in water and gargled }\end{array}$ & $\begin{array}{l}\text { Common colds/cough/ } \\
\text { sore throat }\end{array}$ \\
\hline
\end{tabular}


Table 1 List of the various Herbal plants used by herbalistsand corresponding uses (Continued)

\begin{tabular}{|c|c|c|c|c|c|c|c|c|c|}
\hline $\begin{array}{l}\text { Warburgia } \\
\text { ugandensis Sprague }\end{array}$ & WBHG/13/109 & Sokwon & $\begin{array}{l}\text { East African green } \\
\text { wood }\end{array}$ & Canellaceae & Tree & $\begin{array}{l}\text { Highland/ } \\
\text { escarpment }\end{array}$ & Leaves & $\begin{array}{l}\text { Soaked in water } \\
\text { and gargled }\end{array}$ & Oral thrush \\
\hline $\begin{array}{l}\text { Withaniasomnifera } \\
\text { (L.) Dunal }\end{array}$ & WBHG/13/054 & Tarkukai & Red Cherry & Solanaceae & Shrub & $\begin{array}{l}\text { Lowland/ } \\
\text { escarpment }\end{array}$ & Roots/leaves & Boiled and administered & Amoebiasis \\
\hline $\begin{array}{l}\text { Withania somnifera } \\
\text { (L.) Dunal }\end{array}$ & WBHG/13/054 & Tarkukai & Red Cherry & Solanaceae & Shrub & $\begin{array}{l}\text { Lowland/ } \\
\text { escarpment }\end{array}$ & Leaves & Crushed and applied & Chronic skin ulcers \\
\hline Ximenia Americana L. & WBHG/13/105 & Kunyotwo & Yellow plum & Olacaceae & Tree & $\begin{array}{l}\text { Lowland/ } \\
\text { escarpment }\end{array}$ & Seeds & Crushed and oil applied & Wounds \\
\hline Ximenia Americana L. & WBHG/13/105 & Kunyotwo & Yellow Plum & Olacaceae & Tree & $\begin{array}{l}\text { Lowland/ } \\
\text { escarpment }\end{array}$ & Seeds & Crushed and oil applied & $\begin{array}{l}\text { Treatment of hides } \\
\text { and skins }\end{array}$ \\
\hline $\begin{array}{l}\text { Zanthoxylum } \\
\text { chalybeum Engl }\end{array}$ & WBHG/13/061 & Kochon & Knob wood & Rutaceae & Tree & $\begin{array}{l}\text { Lowland/ } \\
\text { escarpment }\end{array}$ & Bark/seeds & $\begin{array}{l}\text { Pounded together and } \\
\text { mixed with other herbs }\end{array}$ & Synergistic herb \\
\hline $\begin{array}{l}\text { Zanthoxylum } \\
\text { chalybeum Engl }\end{array}$ & WBHG/13/061 & Kochon & Knob wood & Rutaceae & Tree & $\begin{array}{l}\text { Lowland/ } \\
\text { escarpment }\end{array}$ & Bark/seeds & Boiled or chewed & Amoebiasis \\
\hline $\begin{array}{l}\text { Zanthoxylun } \\
\text { chalybeum Engl }\end{array}$ & WBHG/13/061 & Kochon & Knob wood & Rutaceae & & $\begin{array}{l}\text { Lowland/ } \\
\text { escarpment }\end{array}$ & Bark & Boiled and administered & Malaria like symptoms \\
\hline $\begin{array}{l}\text { Zanthoxylun } \\
\text { chalybeum Engl }\end{array}$ & WBHG/13/061 & Kochon & Knob wood & Rutaceae & Tree & $\begin{array}{l}\text { Lowland/ } \\
\text { escarpment }\end{array}$ & Bark & Boiled and administered & $\begin{array}{l}\text { Administered to } \\
\text { recuperating patients } \\
\text { (after surgery) }\end{array}$ \\
\hline $\begin{array}{l}\text { Zanthoxylun } \\
\text { chalybeum Engl }\end{array}$ & WBHG/13/061 & Kochon & Knob wood & Rutaceae & Tree & $\begin{array}{l}\text { Lowland/ } \\
\text { escarpment }\end{array}$ & Higher parasites & Burnt and ashes licked & Rheumatism \\
\hline $\begin{array}{l}\text { Zehneria scabra } \\
\text { (Linn f.) Sond }\end{array}$ & WBHG/13/097 & Cheserya & Not found & Cucurbitaceae & $\begin{array}{l}\text { Shrub/ } \\
\text { climber }\end{array}$ & Highland & Leaves & Crushed and administered & Common colds/cough \\
\hline $\begin{array}{l}\text { Zehneria scabra } \\
\text { (Linn f.) Sond }\end{array}$ & WBHG/13/097 & Cheserya & Not found & Cucurbitaceae & $\begin{array}{l}\text { Shrub/ } \\
\text { climber }\end{array}$ & Highland & Whole plant & Boiled with other herbs & Cancer \\
\hline $\begin{array}{l}\text { Zehneria scabra } \\
\text { (Linn f.) Sond }\end{array}$ & WBHG/13/097 & Cheserya & Not found & Cucurbitaceae & $\begin{array}{l}\text { Shrub/ } \\
\text { climber }\end{array}$ & Highland & Whole plant & Boiled and administered & $\begin{array}{l}\text { Administered to } \\
\text { recuperating patients }\end{array}$ \\
\hline $\begin{array}{l}\text { Ziziphus mauritania } \\
\text { var. spinachristi } \\
\text { (L.) Wild. }\end{array}$ & WBHG/13/100 & Tilomwo & Christ thorn/jujube & Rhamnaceae & Tree & Lowland & Bark & Boiled with water & Herbal "tea" \\
\hline $\begin{array}{l}\text { Ziziphus mauritiana } \\
\text { var. spina Christi } \\
\text { (L.) Willd }\end{array}$ & WBHG/13/100 & Tilomwo & Christ thorn/jujube & Rhamnaceae & Tree & $\begin{array}{l}\text { Lowland/ } \\
\text { escarpment }\end{array}$ & Bark & Chewed raw & Abdominal (colic) pains \\
\hline Unidentified & WBHG/13/015 & $\begin{array}{l}\text { Mindililwo } \\
\text { ne mining }\end{array}$ & & & Herb & Escarpment & Leaves & Smoked & Drug of abuse \\
\hline Unidentified & WBHG/13/048 & Seremwo & & & Tree & Escarpment & Bark & Boiled and administered & Appetizer \\
\hline Unidentified & WBHG/13/057 & Turesio & & & Tree & Highland & Bark/roots & Burnt and with other herbs & Cancer \\
\hline Unidentified & WBHG/13/057 & Turesio & & & Tree & Highland & Leaves & Burnt and ashes licked & "Kipei" condition \\
\hline
\end{tabular}


Table 2 Diversity of medicinal plant use

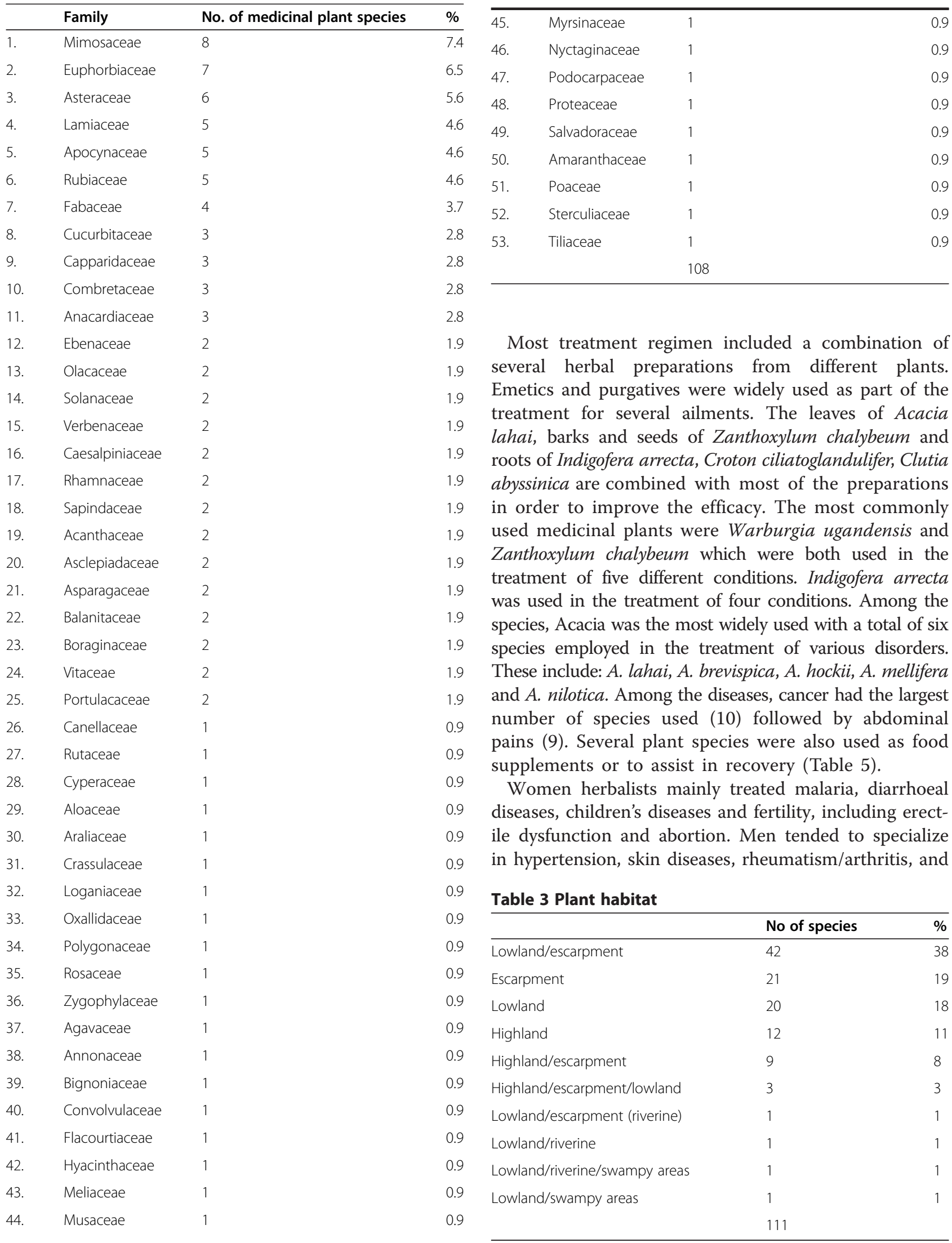

Table 2 Diversity of medicinal plant use (Continued)

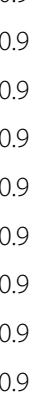
. 
Table 4 Plant habit

\begin{tabular}{lll}
\hline Type & No of species & \% \\
\hline Tree & 41 & 37 \\
Herb & 32 & 29 \\
Shrub & 29 & 26 \\
Liana/climber & 7 & 6 \\
Shrub/climber & 2 & 2 \\
& 111 & \\
\hline
\end{tabular}

surgical procedures including craniotomy, removal of ganglions and setting fractures. The most prevalent infections on the highland areas were respiratory diseases mainly; common colds, flu, pneumonia, upper respiratory tract infections and allergies due to cold weather. Malaria and animal diseases are more prevalent in the lowlands areas. Other common diseases in the district include diarroheal diseases, skin diseases, eye diseases and urinary tract infections. The plants used by the herbalists in the two regions correlated to the prevalent diseases. For instance, common colds and flu were treated by highland plants such as Schefflera volkensii, Zehneria scabra and Nuxia congesta; whereas malaria was treated by either lowland or escarpment plants such as Terminalia spinosa, Zanthoxylun chalybeum, Pentas longiflora and Diospyros scabra (Table 1).

\section{Herbal preparations}

The preparations consisted of roots, barks, leaves, twigs, sap and fruits and were prepared in different forms depending on the intended medicinal use. The proportions of the parts used are as illustrated in Table 6. Leaves were the most widely used (30\%), followed by bark $(21 \%)$ roots $(16 \%)$, fruits $(8 \%)$, whole plant $(7 \%)$, seeds $(5 \%)$, tubers $(5 \%)$, fruit bodies $(3 \%)$, higher parasites $(2 \%)$ and flowers $(2 \%)$.

\section{Decoctions}

The plant parts are boiled or simply soaked in water and the decoction taken alone, or in some instances combined with honey, soup, or milk if the decoction is from a bitter plant. The soup is made from the head, intestines and hooves of an animal, preferably a goat or cow. A mixture of barks, leaves and fruits from several herbs may also be used depending on the condition being treated and the concoction administered to the patient.

\section{Ashes}

Leaves are dried and burnt to form powder ash locally referred to as "Tusan" in Marakwet. The ashes may then be licked, or in some instances applied on incisions that are made on the skin to treat particular ailments.

\section{Green leaves}

The leaves are crushed, and sometimes soaked in water and the resultant concoction may be drunk, or applied directly on the affected area such as in the treatment of toothache. The latex may also be applied on the affected area of the skin, an example being in the treatment of allergy.

\section{Others}

Higher parasites [32], "Sagorket" and fruit bodies of fungi "Lobchon" that grow on trees (Sporocarps) are also used.

\section{Plant use}

\section{Emetics}

There are several plants that are used as emetics. The fruits of the bitter gourd, Cucumis dipsaceus are crushed and administered to the patient to induce vomiting. The fruits of Gardenia spp. are also crushed and given to the patient to induce both vomiting and diarrhea. The concoction is considered to be dangerous on overdose, and is therefore only administered by an experienced herbalist. Others used include the leaves of Calotropis procera and roots of Vernonia brachycalyx which are boiled and the decoctions administered.

\section{Purgatives}

The roots of Croton megalocarpus are boiled and the decoction used as a purgative. Others include the barks from Pavetta abyssinica and Ekebergia rueppelliana.

\section{Heart burn}

The treatment involves licking of the ashes prepared from the leaves and twigs of Cirsium vulgare. An alternative treatment involves the administration of decoction from the boiled roots of Croton ciliatoglandulifer. The leaves of Dactyloctenium aegyptium are also crushed and administered to patient. Similarly, the leaves of Capparis cartilaginea are chewed for the relief of both heartburn and treatment of peptic ulcers. We interviewed two patients who informed us that upon chewing the leaves, they felt as though "smoke came out of their nose", before getting some relief. The ash of Bryophyta spp. and that obtained from burning the tip end of the fruit of Ensete ventricosum are also licked.

\section{Stomachache}

The tuber of Cyperus esculentus is chewed and swallowed. The roots of Indigoferra arrecta and bark of Ehretia cymose var. silvatica are boiled and decoction taken to relieve stomachache. Alternative cure involves chewing of the leaves of Warburgia ugandensis. The roots of Saba comorensis and the bark of Sclerocarya birrea are boiled and administered to the patient. In the treatment of the 
Table 5 Medicinal plant uses

\begin{tabular}{|c|c|c|}
\hline & No of species used & Percentage \\
\hline Cancer & 10 & 7.1 \\
\hline Abdominal (colic pains) & 9 & 6.4 \\
\hline Food supplements & 5 & 3.6 \\
\hline Heartburns & 5 & 3.6 \\
\hline Herbs used by convalescing patients & 5 & 3.6 \\
\hline Synergistic herbs & 5 & 3.6 \\
\hline Amoebiasis & 4 & 2.9 \\
\hline Emetics & 4 & 2.9 \\
\hline Hypertension & 4 & 2.9 \\
\hline Malaria like symptoms & 4 & 2.9 \\
\hline Purgatives & 4 & 2.9 \\
\hline Stomachache & 4 & 2.9 \\
\hline Toothache & 4 & 2.9 \\
\hline Abortifacients & 3 & 2.1 \\
\hline Antivenom & 3 & 2.1 \\
\hline Colic pain in children & 3 & 2.1 \\
\hline Erectile dysfunction & 3 & 2.1 \\
\hline Eye ailments & 3 & 2.1 \\
\hline Food flavours & 3 & 2.1 \\
\hline Herbal tea & 3 & 2.1 \\
\hline Oral thrush & 3 & 2.1 \\
\hline Removal of ganglions & 3 & 2.1 \\
\hline "Kipei" condition & 2 & 1.4 \\
\hline Administered after surgery & 2 & 1.4 \\
\hline Arthritis & 2 & 1.4 \\
\hline Chest congestion (wheezing) & 2 & 1.4 \\
\hline Common colds/cough & 2 & 1.4 \\
\hline Craniotomy (surgery) & 2 & 1.4 \\
\hline Diabetes & 2 & 1.4 \\
\hline $\begin{array}{l}\text { Enhance lactation in breastfeeding } \\
\text { mothers }\end{array}$ & 2 & 1.4 \\
\hline Itchy skin rashes & 2 & 1.4 \\
\hline Rheumatism & 2 & 1.4 \\
\hline Sexually transmitted diseases & 2 & 1.4 \\
\hline Wounds & 2 & 1.4 \\
\hline Anaemia & 1 & 0.7 \\
\hline Antihaemorrhagic (arrests bleeding) & 1 & 0.7 \\
\hline Appetizers & 1 & 0.7 \\
\hline Blocked nose & 1 & 0.7 \\
\hline Chronic skin ulcers & 1 & 0.7 \\
\hline Common colds/cough/sore throat & 1 & 0.7 \\
\hline Diagnosis of an enlarged prostate & 1 & 0.7 \\
\hline Enlarged prostate & 1 & 0.7 \\
\hline Fever in children & 1 & 0.7 \\
\hline
\end{tabular}

Table 5 Medicinal plant uses (Continued)

\begin{tabular}{lll}
\hline Flu & 1 & 0.7 \\
General Malaise & 1 & 0.7 \\
Headache & 1 & 0.7 \\
Jaundice & 1 & 0.7 \\
Mumps & 1 & 0.7 \\
Obesity & 1 & 0.7 \\
Peptic ulcers & 1 & 0.7 \\
Period pains & 1 & 0.7 \\
Poultice & 1 & 0.7 \\
Removal of retained placenta & 1 & 0.7 \\
Stomachache resulting from & 1 & 0.7 \\
food poisoning & & \\
Teething in children & 1 & 0.7 \\
Treatment of heavy snoring & 1 & 0.7 \\
\hline
\end{tabular}

stomachache resulting food poisoning, the roots of Sphaeranthus ukambensis are chewed or boiled.

Abdominal pains (colic pains)

Treatment involves the boiling of the bark of Acacia hockii and the roots of Nuxia congesta. The tuber of Cyperus esculentus and bark of Acacia nilotica are also chewed to relieve the colic pain. Alternative treatment involves the chewing of the roots of Solanum incanum and Croton ciliatoglandulifer. The barks of Ziziphus mauritiana var spina Christi and Terminalia brownie may either be chewed or boiled to treat the condition. The leaves of Justica spp. may also be used.

\section{Colic pain in children}

The roots and leaves of Hoslundia opposite or tuber of Cyperus esculentus are crushed or chewed and administered to the children. An alternative treatment involves the administration of chewed leaves or decoction from boiled roots of Solanum incanum.

Table 6 Proportions of plant parts used

\begin{tabular}{ll}
\hline Part & $\%$ \\
\hline Leaves & 30 \\
Bark & 21 \\
Roots & 16 \\
Fruits & 8 \\
Whole plant & 7 \\
Seeds & 5 \\
Tubers & 5 \\
Fruit bodies & 3 \\
Higher parasites & 2 \\
Flowers & 2 \\
\hline
\end{tabular}




\section{Amoebiasis}

A concoction made from the boiled roots and leaves of both Hoslundia opposite and Withania somnifera leaves of Lippia javanica and the bark and seeds of Zanthoxylun chalybeum.

\section{Kipei condition}

This condition manifests with oral thrush and severe abdominal pain. It is treated by using the ashes made from higher parasites [32], "Sagorket" that grow on Croton dichogamus which are licked. The leaves of Clerodendrum myricoides and "Turesio" are also burnt and ashes licked.

\section{Period pains}

The bark of Faurea saligna is boiled and concoction administered.

\section{Headache}

The bark of Warburgia ugandensis is burnt and smoke sniffed.

\section{Toothache}

The leaves of Fuerstia africana and/or roots of Indigoferra arrecta are crushed and applied on the affected tooth. The leaves of Warburgia ugandensis may also be used. An alternative treatment involves the application of the whole the crushed Spilanthes mauritiana plant on the site.

\section{Teething in children}

The ashes of the Bryophyta spp.are rubbed on the gums to relive the pain and soreness associated with teething.

\section{Malaria and fever}

The bark of Terminalia spinosa alone, or in combination with that of Zanthoxylun chalybeum are boiled and the concoction administered. The fruits and bark of Pentas longiflora may also be added. To reduce the fever associated with malaria especially in young children, the bark Diospyros scabra is boiled and the patient is bathed in the concoction.

\section{Jaundice}

Treatment involves the chewing of the bark of Terminalia brownii or administration of the decoction obtained by the boiling of the bark of the same plant.

\section{Common colds and cough}

The decoction from boiled roots of Uvaria sp var. scheffleri plant is combined with leaves of either Zehneria scabra or Warburgia ugandensis which are chewed for treatment of colds including sore throats. The boiled roots of Nuxia congesta are used in the treatment of flu. The dried resin ("Manga") of Schefflera volkensii is inhaled to clear blocked nose.

\section{Chest congestion (wheezing)}

The whole of Caralluma acutangula is crushed and administered, especially children. An alternative treatment involves use of a concoction from the roots, bark and flowers of Croton dichogamus are boiled and administered.

\section{Oral thrush}

The Whole of crushed Spilanthes mauritiana is chewed. For oral thrush in children, they are crushed and mixed with water or milk and administered. Alternative treatment involves gargling of the crushed leaves of Warburgia ugandensis or Hoslundia opposite.

\section{Eye ailments}

The leaves of Ocimum basilicum are crushed and concoction applied to the eye. The leaves Fuerstia Africana of are also crushed and instilled on the affected eye. An alternative treatment involves the grinding the dried barks of either Olea Africana and applying the powder into the affected eye. The powder from Podocarpus gracilior may also be used.

\section{Wounds}

The seeds of Ximenia Americana are crushed and the oil extracted applied on the wounds. Alternatively, the leaves of Aloe spp. are broken and the sap squeezed on to the exposed wounds. The leaves of either Capparis cartilaginea or Indigofera arrecta are used in the treatment of skin sores and ulcers; whereas those of Withania somnifera are used in treatment of chronic skin ulcers. Aloe spp. was reportedly used for the treatment of wounds in Nandi and ulcers in Sabaot [20,21].

\section{Cancer}

Ashes from burnt fruit bodies of fungi "Lobchon" that grow on trees (Sporocarps) such as Acacia spp., Faidherbia albida, Combretum apiculatum, Albizia species and Pappea capensis are mixed with milk and administered to the patient. The roots of Indigofera arrecta may also be boiled or chewed and combined with the herbal concoction. Drimia indica and Albuca bracteata are also used in the treatment of cancer and may be combined with the other herbs. An alternative treatment involves the use of Zehneria scabra. A mixture of the whole plant, together with the bark and roots of "Turesio", one maize cob and bean husks are burnt. The resultant ashes are then mixed with ground finger millet and administered. The whole crushed plant of Portulaca oleracea may also be used. Researchers from previous studies have reported cytotoxic effects from the isolates of Acacia spp. [33], Albizia species [34] and Zehneria scabra [35]. 


\section{Skin rashes}

The crushed leaves of Commicarpus africanus are combined with those of Acacia brevispica and rubbed on affected area. The bark of Olea Africana may also be boiled and the decoction taken to treat the itchy rashes.

\section{Removal of ganglions}

The leaves of Acacia brevispica are crushed and mixed with those of Commicarpus africanus and rubbed on the area of the body with a ganglion cyst. It is repeated until the ganglion disappears. We interviewed one patient who informed us that the ganglion disappeared completely after applying the preparation for three days.

\section{Herbs used during craniotomy}

The whole of Spilanthes mauritiana is crushed and soaked in water. It is then administered to patient on the eve of surgery, and for the next two days after surgery. The sap of Aloe $s p$. is then applied on the wound to reduce infection. In order to enhance recovery, the bark of Zanthoxylun chalybeum and the whole of Zehneria scabra plant are crushed and the resultant concoction administered to the patient.

\section{Sexually transmitted diseases}

Acacia melifera is used as an antibiotic for the treatment of venereal diseases. The roots are boiled and the decoction administered to the patient. It is combined with the whole of Ipomoea lapidosa plant and roots of Berchemia discolor.

\section{Hypertension}

The bark of Prunus Africana mixed with that of Podocarpus graciliar are boiled and administered to the patient in the treatment of hypertension. The concoction made from the tuber of Rumex acetosella may also be used.

\section{Diabetes}

The tubers of Rumex acetosella are boiled alone, or in combination with the bark of Sclerocarya birrea and the decoction administered.

\section{Obesity}

The seeds of Boscia coriacea are boiled and administered to the patient.

\section{Arthritis}

The tubers of Ornithogalum tenuifolium are crushed and applied on the affected knees or joints. The roots of Crassocephalum mannii are boiled and administered.

\section{Rheumatism}

Higher plant parasites that grow on the following trees; Acacia mellifera, Zanthoxylun chalybeum and Pappea capensis are burnt and the ashes licked.

\section{Poultice}

The leaves of Kalanchoe germanae are pound and rubbed on the painful areas of the body. In the Sabaot study, Kalanchoe mitejea is used [21].

\section{Erectile dysfunction}

The roots of Berchemia discolor mixed with those of Clutia abysinica are boiled and the concoction drunk to improve virility. An alternative treatment involves boiling the concoction from the bark and chewing the roasted seeds of Sterculia aficana. Young shoots of the whole plant of Tribulus terrestris may also be chewed.

\section{Renal disorders/enlarged prostate}

The fruits of Saba comorensis are used in the diagnosis of renal disorders including an enlarged prostate. It is also used in the diagnosis of sexual function, to ascertain whether the man is responsible in families who cannot bear children. The herbalists informed us that it was difficult to obtain the fruits as the plant has yellow sweet fruits which are a delicacy for baboons. The plant is used only in men, and appears to have some diuretic activity. In order to make a diagnosis of a renal disorder, the patient is given the fruits, and if the patient does not pass yellow urine after about one hour, then it is assumed that he has a renal disorder especially an enlarged prostate, or a venereal disease. It is also interpreted to mean that he has erectile dysfunction and therefore sterile, if they have been in a marriage and have not been able to get children. A concoction made from the bark of Prunus africana is also used on the treatment of an enlarged prostate.

\section{Mumps}

The barks of Erythrina abyssinica are used in the treatment of mumps. They are boiled and concoction administered.

\section{Lactation}

In order to enhance lactation in breastfeeding mothers, the leaves of Dolichos spp are used. They are dried, crushed and dissolved in water before administering to the mother. An alternative preparation consists of the use of crushed leaves and twigs of Lantana trifolia.

\section{Abortifacients}

The bark of Albizia spp is boiled and administered to induce abortion. It is also used as a contraceptive. The leaves and seeds of Cassia occidentalis/obtusifolia and 
stem of Cyphostemma serpens are also used as abortifacients. They are crushed and soaked in water before administration.

\section{Removal of placenta}

In case of a retained placenta in both humans and animals, the bark of Heeria reticulata is crushed, dissolved in water and administered.

\section{Antihaemorrhagic}

The bark of Cassia didymobotrya is used to stop bleeding. The dried bark is ground and powder applied on the bleeding area.

\section{Anaemia}

Iron defiency anaemia is treated by supplementing food with Crotalaria brevidens.

\section{Heavy snoring}

The leaves of Coccinia grandis are used as a remedy to treat people who snore heavily. Patients are advised to use the leaves as vegetables.

\section{Astringent}

The leaves and twigs of Lantana trifolia are used as astringents.

\section{General malaise/appetizers}

The roots of Carissa edulis are boiled and decoction administered to the patient with general malaise symptoms. Ripe fruits of the same plant are used as appetizers. The bark of "Seremwo" is also boiled and administered to patient for the same purpose.

\section{Natural healing vegetables and fruits}

There are some specific vegetables that are recommended for use by convalescing patients or those in frail health. After a course of treatment, the spider plant Gynandropsis gynandra is cooked and milk added. It is then administered to the patients in order to speed up the recovery. Other healing vegetables include the leaves of Digera muricata, Crotalaria incana and Leptadenia hastate. It also includes the tender young plant of Tribulus terrestris, the whole of Dactyloctenium aegyptium and Portulca qudrifida plants, as well as the leaves and oil from the seeds of Balanites aegyptiaca. The fruits of Vangueria madagascariensis and Vangueria apiculata are used by recuperating patients.

\section{Food flavours}

The leaves of Lippia javanica are used. There are three species of this plant used, but only one is used for this purpose and is identifiable by experienced herbalists. The plant is a potent sweetener and is boiled with food, especially maize in order to improve the taste. The flowers of Capparis cartilaginea are also used, especially in the preparation of fish stew to reduce the smell as fish is not generally popular among the Kalenjin community.

\section{Herbal "tea"}

The barks of Ziziphus Mauritania var. spina Christi and Acacia hockii are boiled in water used as herbal beverages in a similar way as tea leaves. The bark of Psiadia Arabica may also be used.

\section{Scorpion, bee, and wasp stings}

The leaves of Acalypha fruticosa are applied directly on the stung area of the body.

\section{Antivenom}

The bark of Euclea divinorum and roots of Tragia brevipes are used as antivenom. They are crushed and the resultant preparation applied into an incision made on the area that has been bitten by the snake. The juice from the fruits of Solanum incanum combined with the crushed bark of Lannea fulva are then applied on the bitten area. The sap from the leaves of Sansevieria intermedia are used to kill snakes. The leaves are squeezed and juice applied on the opening of the hole where snakes reside. The authors in the Sabaot study also reported the use of Euclea divinorum for the treatment of snake bites [21].

\section{Talisman}

It is a common practice to make three incisions on the temples, nape and epigastric region among the Marakwet and Pokot tribes in children or a person who has been ill for a long time in order to protect them from witches or people with "bad eyes" (evil intentions) or evil influences [13]. The burnt ashes of Cirsium vulgare are used for this purpose. The tuber of Cyperus esculentus is also chewed directly and the saliva smeared on children where it acts as a talisman.

\section{Drug of abuse}

"Mindililwo ne mining", is an ephemeral plant that is usually chewed to provide a sweet taste, but the elders informed us that they know it as a drug of abuse when the leaves are smoked. In order to discourage children from going near the plant; they are informed that the plant only grows near where "Ilat" lives; and that it is "nguekab chesowiloy" (a vegetable that belongs to the devil). "Ilat" is considered an agent of "Asis", the Supreme Being, and omnipotent arbiter of all things and guarantor of right. "Ilat", in mundane terms "thunder and lightning" is dreaded, for he can cause death. He is invincible, and is seen when lightning strikes something, such as a house or a tree. He acts in the interest of justice and may strike an offenders house or stock [13]. 


\section{Ceremonial}

Among the Marakwet, and indeed all Kalenjin communities, Periploca linearifolia is considered a sacred plant. It is used in all celebration ceremonies including weddings and initiations $[20,21,36]$.

\section{Perfumes}

The leaves of Iboza spp. are crushed and applied to the body as perfume. The dried resin obtained from Schefflera volkensii is added to tobacco to improve the aroma and can also be applied on the body.

\section{Fermentation}

The fruits of Kigelia Africana and Flacourtia indica, and the leaves of Grewia villosa and Croton dichogamus are used to provide fermentation during the brewing of traditional beer.

\section{Hides and skins}

The seeds of Ximenia americana and castor plant are used for the treatment of hides and skins.

\section{Toothbrush}

The twigs of the following tree species are used as toothbrushes: Salvadora persica, Dodonaea viscosa, Diospyros spp., Cordia sinensis, Euclea divinorum and Grewia bicolor.

\section{Arrow poison}

Acokanthera schimperi is used as an arrow poison. The roots are boiled and left to settle and after evaporation, the concentrate applied on the arrows. The efficacy of the poison is then tested in dogs. In order to enhance the potency, the poison is mixed with bile from either a crocodile or monitor lizard. The plant has been used as an arrow poison in many parts of Africa [37-39]. However, in the Nandi study Acokanthera schimperi was reportedly used as an antibiotic for the treatment of syphilis [20].

\section{Pesticides}

The tuber of Cyphostemma cyphopetalum is used to eradicate all insects. It is crushed water added, and the resultant concoction is used against all insects. When used to kill household insects like mites, the concoction is left standing in the room for one day and after that left for at least two before occupation since the plant is very poisonous. It is also employed as a poison to kill unwanted animals. The leaves and fruits of Periploca linearifolia are used for the eradication of lice in goats and sheep. The whole plant is crushed and soaked in water and applied on the skin of the animals. It is also used as a poison. The fruits of Balanites rotundifolia are also employed as pesticides. They are crushed, soaked in water and used against insect pests in both crops and animals. The leaves and twigs of Hypoestes forskaolii are crushed and used to kill pests especially lice on domestic animals.

\section{Veterinary uses \\ Poisoning/constipation}

The whole of Maesa lanceolata plant is crushed and after the addition of a little water administered to an animal which has eaten poison or constipated to induce diarrhoea. Alternatively, the leaves of Heeria reticulata are crushed and administered.

Our biggest challenge was to gain the trust from the herbalists. It took a lot of persuasion and involvement of opinion leaders to let them understand our intentions and volunteer the information. Some conditions described by the herbalists were not easily identifiable as we could not access the patients, or they were already healed by the time we met them. Herbalists from the same area tended to use the similar herbs for the same conditions. However, we found many incidences of herbalists from a similar location using the same plants for totally different conditions, especially those widely known to specialize in the treatment of a specific disorder such as infertility. This could be attributed to the fact that the traditional knowledge is by and large a closely guarded family secret [7].

Emetics and purgatives were widely used. Among the Kalenjin community, emesis "Ketap" and diarrhoea "Kegoor" constituted important forms of treatment as they were believed to decontaminate the body from pollutants, just like their Maasai counterparts [40,41]. It was therefore important for each person to use either purgatives, emetics or in most instances both at least once a year in order to cleanse the system so as to maintain good health; or to prevent a disease condition that they referred to as "Ngwono". The term "Ngwono" is also separately a synonym for poison. "Ngwono" was a very prominent condition that was mentioned by the herbalists. They described the condition as presenting with malaria like symptoms including malaise, fatigue, lack of appetite, nausea and vomiting, including the vomiting of a yellow substance; presumably bile. The condition according to the herbalists could only be treated by inducing vomiting and diarrhoea by use of emetics and purgatives. Both classes of herbal drugs were also taken in preparation of major events such as festive seasons. In fact, emesis and diarrhoea were mandatory for boys or girls as part of the preparations, weeks before undergoing circumcision. However, the herbalists informed us that some of the emetics and purgatives such as Gardenia Spp. were quite toxic on overdose and could only be administered by experienced herbalists. Calotropis procera in large doses is known to be an emetic and a purgative $[42,43]$. Croton megalocarpus 
has also been used as a purgative and Pavetta abyssinica for indigestion [18].

Our research team could not quite figure out the condition that most of the herbalists and patients we interviewed referred to as cancer, "Seryan". The symptoms they described appeared to suggest myasis, chronic tropical ulcer, or probably squamous cell carcinoma. They described cancer as a condition that manifests with a chronic wound on the skin. After treatment using the cancer specific herbs for some period of time, the area of the skin with the ulcer reduced, finally into a small size which they described as the mouth, "Kuti". Upon pressing the areas around the "mouth", a substance resembling a pupa emerging from a cocoon which they referred to as "Kutyan" appears, indicating that the patient was now healed. This could also suggest guinea worm infestation although unlikely as this is not a Guinea worm endemic area. We concluded that this was most likely thick solid pus from an abscess, suggesting involvement of some infection. Had we seen a patient with the condition before treatment, then perhaps we would have made a diagnosis. However, the fact that some of the plants used in the treatment of the condition such as Acacia spp., Albizia species and Zehneria scabra have been shown to possess some cytotoxic activities may actually suggest that it is actually cancer [33-35]. Indeed cancer was the condition treated by the highest number of species, indicating the complexity of the condition (Table 5). "Kipei" was another condition that was not very clear. The herbalists described the condition as that presenting with severe abdominal pain and oral thrush, suggestive of some form of immunosuppression. Likewise, we were not able to see a patient with this condition. However, we managed to interview some other patients with other conditions, such as ulcers and ganglion growths.

Despite the long distance between Marakwet and Sabaot districts (about 200 kilometers away), there were 24 recorded plant species that were used in both communities, with 9 of them having similar uses. These include: Acacia lahai, Albezia spp., Aloe spp., Carissa edulis, Clerodendrum myricoides, Diospyros spp., Dolichos spp., Ensete ventricosum, Erythrina abyssinica, Euclea divinorum, Flacourtia indica, Indigofera arrecta, Kalanchoe germanae, Lantana trifolia, Maesa lanceolata, Periploca linearifolia, Prunus Africana, Ricinus communis, Schefflera volkensii, Cassia (Senna) didymobotrya, Solanum incanum, Vangueria apiculata, Warburgia ugandensis, Withania somnifera [21]. The nine plants used for similar indications were: Aloe spp. (wounds/ulcers), Dolichos spp. (enhance lactation/fertility), Euclea divinorum (antivenom), Kalanchoe spp. (poultice), Periploca linearifolia (ceremonial/ initiation), Prunus Africana (UTIs), Schefflera volkensii (respiratory disorders), Solanum incanum (abdominal pains) and Warburgia ugandensis (headache).
The species that were common with Nandi were (eight) including: Acokanthera schimperi, Aloe spp., Carissa edulis, Justica spp., Cassia (Senna) didymobotrya, Ehretia cymose, Justica spp., Kigelia Africana, Periploca linearifolia, with four having similar indications [20]. The 4 plants with similar indications were: Aloe spp.(wounds), Ehretia cymose (stomachache/typhoid), Justica spp. (abdominal pains/ulcers), Periploca linearifolia (ceremonial/ rituals). Four plants; Aloe spp., Carissa edulis, Cassia (Senna) didymobotrya, and Periploca linearifolia were commonly used in all the three districts; with Aloe spp. and Periploca linearifolia having similar uses in all the three districts.

\section{Conclusion}

The study provides a comprehensive report on the vast wealth of traditional medical knowledge, health practices and plant use among the Marakwet community. Scientific evaluation of the medicinal plants may lead to the development of new drugs. There are few records on traditional medicinal plant usage among the various communities in Kenya despite their widespread use. There is therefore urgent need to document this information, as it is rapidly disappearing due to influence of western medicine and other reasons including socio-cultural issues and overexploitation coupled with rapid deforestation. It is important to collect this information and develop a database of medicinal plants for future research and potential development of new drugs.

\section{Competing interests}

The author's declare that they have no competing interests.

\section{Authors' contributions}

All the authors shared the contributions to the work fieldwork of the manuscript. Kipkore and Wanjohi identified the plants. Kigen and Rono interviewed the herbalists and patients, in order to identify the illnesses. Kipkore and Kigen analyzed the data and prepared the manuscript. All authors read and approved the final manuscript.

\section{Author details}

${ }^{1}$ Department of Forestry, University of Eldoret, P.O. Box 1125, Eldoret, Kenya. ${ }^{2}$ Department of Wildlife Management, University of Eldoret, P.O. Box 1125 Eldoret, Kenya. ${ }^{3}$ Ophthalmologist, Kitale Hospital and North Rift Zonal eye surgeon; Ministry of Health, P.O. Box 98, Kitale, Kenya. ${ }^{4}$ Department of Pharmacology and Toxicology, Moi University School of Medicine, P.O. Box 4606, Eldoret, Kenya.

Received: 30 May 2013 Accepted: 22 January 2014

Published: 20 February 2014

\section{References}

1. Balunas MJ, Kinghorn AD: Drug discovery from medicinal plants. Life Sci 2005, 78(5):431-441.

2. Torres MP, Rachagani S, Purohit V, Pandey P, Joshi S, Moore ED, Johansson SL, Singh PK, Ganti AK, Batra SK: Graviola: a novel promising natural-derived drug that inhibits tumorigenicity and metastasis of pancreatic cancer cells in vitro and in vivo through altering cell metabolism. Cancer Lett 2012, 323(1):29-40.

3. Vickers A, Zollman C, Lee R: Herbal medicine. West J Med 2001, 175(2):125-128.

4. WHO: Traditional Medicine, Fact sheet; 2008. http://www.who.int/mediacentre/ factsheets/fs134/en/. 
5. WHO: WHO Traditional Medicine Strategy 2002-2005. Geneva: WHO; 2002. www.who.int/topics/traditional_medicine/en/; whqlibdoc.who.int/hq/2002/ who_edm_trm_2002.1.pdf. Accesed on 19/112/2011.

6. Nagata JM, Jew AR, Kimeu JM, Salmen CR, Bukusi EA, Cohen CR: Medical pluralism on Mfangano Island: use of medicinal plants among persons living with HIV/AIDS in Suba District, Kenya. J Ethnopharmacol 2011, 135(2):501-509.

7. Kigen GK, Ronoh HK, Kipkore WK JKR: Current trends of traditional herbal medicine practice in Kenya: a review. Afr J Pharmacol Ther 2013, 2(1):32-37.

8. Rukangira E: Medicinal Plants and Traditional Medicine in Africa:Constraints and Challenges. Nairobi, Kenya: Conserve Africa International; 2001. (www.conserveafrica.org.uk/), adapted on 16/11/2011.

9. Beardsley N: The Kalenjins: a look at why they are so good at long-distance running. 2005. http://anthropology.ua.edu/bindon/ant475/ Papers/Beardsley.pdf. Accessed on 25/01/2014.

10. Fisher M: Why Kenyans Make Such Great Runners: A Story of Genes and Cultures: How an ethnic minority that makes up $0.06 \%$ of the world's population came to dominate most of its long-distance races; 2012. http:// www.theatlantic.com/international/archive/2012/04/why-kenyans-makesuch-great-runners-a-story-of-genes-and-cultures/256015/. Accessed on 29/08/2013.

11. AIPS Magazine: How Ugandan Kiprotich Ruined Kenya's Marathon Salvage Plot. London: International Sports Press Association; 2012. http://www. aipsmedia.com/index.php?page $=$ news $\&$ cod $=8568 \& \mathrm{tp}=\mathrm{n}$ : Accessed on 25/01/2014.

12. Orville BJ: The Kalenjin of Kenya; 1996. http://dickinsg.intrasun.tenj.edu/ nations/kenya/kalenjin.html. Accessed on 27/08/2013.

13. Kipkorir BE, Welbourn F: The Marakwet of Kenya. In A Preliminary Study, By B. E. Chapter 2nd edition. E.A.L.Bureau: Kipkorir, Frederick Burkewood Welbourn; 2008:8-14.

14. Nyamwaya D: African Indigenous Medicine. Nairobi: KEMRI; 1992.

15. Sindiga INCC, Kanunah MP: Traditional Medicine in Africa, East African Publishers; Chapter 3; 1995. http://books.google.co.ke/books/about/ Traditional_Medicine_in_Africa.html: Accesed on 31/07/2012.

16. Lindsay RS, Hepper FN: Medicinal plants of Marakwet, Kenya. Kew, Richmond, United Kingdom: Royal Botanic Gardens; 1978:49. Lippold, H., 1980.

17. Thairu K: The African Civilization. Nairobi: Oxford University Press; 1975.

18. Kokwaro JO: Medicinal Plants of East Africa. 3rd edition. Nairobi: University of Nairobi Press; 2009.

19. Pascaline J, Charles M, George O, Lukhoba C: An inventory of medicinal plants that the people of Nandi use to treat malaria. J Anim Plant Sci 2011, 9:1192-1200

20. Jeruto P, Lukhoba C, Ouma G, Otieno D, Mutai C: An ethnobotanical study of medicinal plants used by the Nandi people in Kenya. J Ethnopharmacol 2008, 116(2):370-376.

21. Okello SV, Nyunja RO, Netondo GW, Onyango JC: Ethnobotanical study of medicinal plants used by Sabaots of Mt. Elgon Kenya. Afr J Tradit Complement Altern Med 2009, 7(1):1-10.

22. USAID/Kenya EMC: Elgeyo Marakwet County - USAID/Kenya; 2011. http:// kenya.usaid.gov/sites/default/files/profiles/Elgeyo\%20Marakwet_Dec2011\% 2021.pdf. Accesed on 23/08/2013.

23. Gunlycke N, Tuomaala A: Detecting forest degradation in Marakwet district. Kenya: Using remote sensing and GIS; 2011.

24. Chebet JG: Forestry resources in Marakwet community. Republic of Kenya: A look at the socioeconomic implication of the fast disappearing of the forestry resources; 2010.

25. Cherangani: BirdLife International (2012) Important Bird Areas factsheet: Cherangani Hills. Birdlife Data Zone 2012. http://www.birdlife.org/datazone/ sitefactsheet.php?id=6433. Accessed on 09/11/2012.

26. Wass P (Ed.): Kenya's Indigenous Forests, Status, Management and Conservation'. Switzerland, and Cambridge, United Kingdom: IUCN, Gland; 1995.

27. WRI: Nature's Benefits in Kenya. An Atlas of Ecosystems and Human Well-Being. Washington D.C. and Nairobi: World Resources Institute; 2007. http://pdf.wri. org/kenya_atlas_fulltext_150.pdf. Accessed on 31/07/2013.

28. Akotsi EFN, Gachanja M, Ndirangu JK: Changes in Forest Cover in Kenya's Five "Water Towers", 2003-2005. Nairobi Kenya: Department of Resource Surveys and Remote Sensing, Kenya Forests Working Group (KFWG); 2006:5-43. http://www.unep.org/dewa/Portals/67/pdf/forest_catchment_2005_report. pdf .accesed on 30/07/2013.
29. IEA: IEA-little-fact-book, the socio economic \& political profiles of Kenya's Districts. 2002. www.ieakenya.or.ke/ .../238-the-little-fact-book-the-socioeconomic. Accessed on 9/3/2013.

30. Beentje HJ: Kenya Trees, Shrubs, and Lianas. Nairobi: National Museums of Kenya; 1994.

31. Agnew ADQ: Upland Kenya Wild Flowers and Fern. 3rd edition. Nairobi: Nature Kenya-The East Africa Natural History Society; 2013.

32. Ellis SD, Boehm MJ, LH R: Parasitic Higher Plants - Ohioline - The Ohio State University. 2008. http://ohioline.osu.edu/hyg-fact/3000/pdf/PP401_09. pdf. Accesed on 17/11/2012.

33. Sakthivel KM, Kannan N, Angeline A, Guruvayoorappan C: Anticancer activity of Acacia nilotica (L.) Wild. Ex. Delile subsp. indica against Dalton's ascitic lymphoma induced solid and ascitic tumor model. Asian Pac J Cancer Prev 2012, 13(8):3989-3995.

34. Cao S, Norris A, Miller JS, Ratovoson F, Razafitsalama J, Andriantsiferana R, Rasamison VE, TenDyke K, Suh T, Kingston DG: Cytotoxic Triterpenoid Saponins of Albizia gummifera from the Madagascar Rain Forest $\nabla, 1$. J nat products 2007, 70(3):361-366.

35. Kongue MD, Talontsi FM, Lamshoft M, Kenla TJ, Dittrich B, Kapche GD, Spiteller M: Sonhafouonic acid, a new cytotoxic and antifungal hopene-triterpenoid from Zehneria scabra camerunensis. Fitoterapia 2013, 85:176-180

36. Langley M: The Nandi of Kenya: Life Crisis Rituals in a Period of Change. New York: St. Martin's Press; 1979.

37. Cassels BK: Analysis of a Maasai arrow poison. J Ethnopharmacol 1985, 14(2-3):273-281

38. Kingdon J, Agwanda B, Kinnaird M, O'Brien T, Holland C, Gheysens T, Boulet-Audet $M$, Vollrath F: A poisonous surprise under the coat of the African crested rat. Proc R Soc B Biol Sci 2012, 279(1729):675-680.

39. Watson RR, Preedy VR: Botanical Medicine in Clinical Practice, pg 7. Cromwell Press: Trowbridge UK; 2008

40. Bussmann RW, Gilbreath GG, Solio J, Lutura M, Lutuluo R, Kunguru K, Wood N, Mathenge SG: Plant use of the Maasai of Sekenani Valley, Maasai Mara, Kenya. J Ethnobiol Ethnomed 2006, 2(1):22.

41. Maundu P, Berger D, ole Saitabau C, Nasieku J, Kipelian M, Mathenge S, Morimoto $Y$, Höft R: Ethnobotany of the Loita Maasai: towards a community management of the forest of the lost child. People Plants Working Paper 8. UNESCO, Paris, France; 2001.

42. Gupta M, Biswas TK, Saha S, Debnsth P: Therapeutic utilization of secretory products of some Indian medicinal plants: a review. Indian J Trad Knowledge 2006, 5(4):569-575.

43. Verma R, Satsangi G, Shrivastava J: Ethno-medicinal profile of different plant parts of clotropis procera (Alt.) R. Br. Ethnobotanical Leaflets 2010, 7:3.

\section{doi:10.1186/1746-4269-10-24}

Cite this article as: Kipkore et al:: A study of the medicinal plants used by the Marakwet Community in Kenya. Journal of Ethnobiology and Ethnomedicine 2014 10:24.

\section{Submit your next manuscript to BioMed Central and take full advantage of:}

- Convenient online submission

- Thorough peer review

- No space constraints or color figure charges

- Immediate publication on acceptance

- Inclusion in PubMed, CAS, Scopus and Google Scholar

- Research which is freely available for redistribution 